TRANSACTIONS OF THE

AMERICAN MATHEMATICAL SOCIETY

Volume 353, Number 7, Pages 2753-2772

S 0002-9947(01)02787-8

Article electronically published on March 15, 2001

\title{
DEGREE-ONE MAPS BETWEEN HYPERBOLIC 3-MANIFOLDS WITH THE SAME VOLUME LIMIT
}

\author{
TERUHIKO SOMA
}

Dedicated to Professor Shin'ichi Suzuki on his sixtieth birthday

\begin{abstract}
Suppose that $f_{n}: M_{n} \longrightarrow N_{n}(n \in \mathbf{N})$ are degree-one maps between closed hyperbolic 3-manifolds with

$$
\lim _{n \rightarrow \infty} \operatorname{Vol}\left(M_{n}\right)=\lim _{n \rightarrow \infty} \operatorname{Vol}\left(N_{n}\right)<\infty .
$$

Then, our main theorem, Theorem 2, shows that, for all but finitely many $n \in \mathbf{N}, f_{n}$ is homotopic to an isometry. A special case of our argument gives a new proof of Gromov-Thurston's rigidity theorem for hyperbolic 3-manifolds without invoking any ergodic theory. An example in $\S 3$ implies that, if the degree of these maps is greater than 1 , the assertion corresponding to our theorem does not hold.
\end{abstract}

In this paper, we will study degree-one maps $f: M \longrightarrow N$ between closed, hyperbolic manifolds. According to a stronger version of Mostow's Rigidity Theorem, Gromov-Thurston's Rigidity Theorem in [20, Chapter 6], the volume $\operatorname{Vol}(N)$ of $N$ is not greater than $\operatorname{Vol}(M)$. Moreover, in the case of dimension $\geq 3$, the equality $\operatorname{Vol}(M)=\operatorname{Vol}(N)$ holds if and only if $f$ is homotopic to an isometry. In any dimension $n$ other than three, H.C. Wang [23] proved that the number of closed, hyperbolic $n$-manifolds with volume less than a given constant is finite up to isometry if $n \geq 4$ and up to homeomorphism if $n=2$. On the other hand, in dimension three, the Hyperbolic Dehn Surgery Theorem [20] implies that there are infinitely many, closed, hyperbolic 3-manifolds with bounded volume. So, we are peculiarly interested in degree-one maps between hyperbolic 3-manifolds. Though this stronger rigidity theorem is powerful, one cannot apply it directly to some of the subjects which are important in the study of degree-one maps between geometric 3-manifolds. We refer to [16], [1], [6, 7], [24] and their references for various results and related topics concerning such subjects.

Consider a $C^{1}$-map $g$ homotopic to a degree-one map $f: M \longrightarrow N$ between closed, hyperbolic 3-manifolds. Let $D g: T(M) \longrightarrow T(N)$ be the derivative of $g$. We may regard that $g$ is close to a local isometry if, for any unit tangent vector $v$ to $M$, the norm $\|D g(v)\|$ is close to 1 . The metrical distortion $\delta(g)$ of $g$ is defined by

$$
\delta(g)=\max \{|\|D g(v)\|-1| ; v \in T(M) \text { with }\|v\|=1\} .
$$

Received by the editors November 12, 1999 and, in revised form, July 10, 2000.

1991 Mathematics Subject Classification. Primary 57M99; Secondary 57M50.

Key words and phrases. Degree-one maps, hyperbolic 3-manifolds, Gromov-Thurston's rigidity theorem. 
Theorem 1 shows that, even if $\operatorname{Vol}(M) / \operatorname{Vol}(N)$ is arbitrarily close to 1 , in general, any $C^{1}$-map homotopic to $f$ is far away from being an isometry.

Theorem 1. Let $\varepsilon>0$ be any positive number. Then, there exist closed, connected, orientable, hyperbolic 3-manifolds $M, N$ with $(1-\varepsilon) \operatorname{Vol}(M) \leq \operatorname{Vol}(N)<\operatorname{Vol}(M)$ and satisfying the following.

(0.1) For any $R>0$, they admit a degree-one map $f_{R}: M \longrightarrow N$ such that the metrical distortion $\delta(g)$ of any $C^{1}-$ map $g: M \longrightarrow N$ homotopic to $f_{R}$ is greater than $R$.

The following is our main theorem, where we consider degree-one maps without fixing domain and target manifolds and present a result contrasting with Theorem 1.

Theorem 2. Let $f_{n}: M_{n} \longrightarrow N_{n}(n \in \mathbf{N})$ be degree-one maps between closed, connected, orientable, hyperbolic 3-manifolds with $\lim _{n \rightarrow \infty} \operatorname{Vol}\left(M_{n}\right)=\lim _{n \rightarrow \infty} \operatorname{Vol}\left(N_{n}\right)$ $<\infty$. Then, for all but finitely many $n \in \mathbf{N}, f_{n}$ is homotopic to an isometry.

The proof of Theorem 2 is done by showing that the restriction $f_{n} \mid M_{n, \operatorname{thick}(\varepsilon)}$ : $M_{n, \operatorname{thick}(\varepsilon)} \longrightarrow N_{n}$ is homotopic to a homeomorphism $j_{n}: M_{n, \operatorname{thick}(\varepsilon)} \longrightarrow N_{n, \operatorname{thick}(\varepsilon)}$ $\subset N_{n}$ for all sufficiently large $n \in \mathbf{N}$. However, the fact that $M_{n, \operatorname{thick}(\varepsilon)}$ and $N_{n, \text { thick }(\varepsilon)}$ have the same topological type seems not to be an immediate consequence from the condition on volume limit. Our proof is based on the argument similar to that in Soma [17, where a certain rigidity theorem for hyperbolic 3manifolds of infinite volume was proved by using the notion of $w$-nets, which consist of regular ideal simplices in $\mathbf{H}^{3}$ (see [17, p. 1238]). In the present case, we will define simplicial honeycombs $\mathcal{H}_{z, t}^{(m)}$ and twins $\mathcal{T}_{z, t}$ consisting of (proper) straight 3 -simplices in $\mathbf{H}^{3}$ well approximated by regular ideal simplices. By using these tools, one can show that not only $M_{n, \operatorname{thick}(\varepsilon)}$ is homeomorphic to $N_{n, \operatorname{thick}(\varepsilon)}$, but also the homeomorphism is realized by a map homotopic to $f_{n} \mid M_{n, \operatorname{thick}(\varepsilon)}$.

In the original proof of Gromov-Thurston's Rigidity Theorem, a degree-one map $f: M \longrightarrow N$ between closed hyperbolic 3 -manifolds is lifted to a map $\tilde{f}: \mathbf{H}^{3} \longrightarrow \mathbf{H}^{3}$ between the universal coverings which is radially extended to a $\pi_{1}(M)$-equivariant measurable map $\tilde{f}_{\infty}: \partial \mathbf{H}^{3} \longrightarrow \partial \mathbf{H}^{3}$. In order to show that the extension is possible, the assumption of the equality $\operatorname{Vol}(M)=\operatorname{Vol}(N)$ was crucial. In the general case without setting this assumption, one cannot invoke the argument in 20] to guarantee the existence of $\widetilde{f}_{\infty}$ even if $\operatorname{Vol}(M)$ and $\operatorname{Vol}(N)$ are arbitrarily close to each other. As a special case of our argument, we will have a new proof of their rigidity theorem without using radially extended maps and hence without relying on any ergodic theory, see Remark 1 in $\S 3$.

When the degree of maps is greater than 1, the assertion corresponding to Theorem 2 does not hold. For any $d_{0} \in \mathbf{N}$ with $d_{0}>1$, there exist degree- $d_{0}$ maps $f_{n}: M_{n} \longrightarrow N_{n}$ between closed, connected, orientable, hyperbolic 3-manifolds with $\lim _{n \rightarrow \infty} \operatorname{Vol}\left(M_{n}\right)=d_{0} \lim _{n \rightarrow \infty} \operatorname{Vol}\left(N_{n}\right)<\infty$, but such that each $f_{n}$ is not homotopic to a (locally isometric) covering, see Example 1 in $\S 3$. Let $\rho_{n}: \pi_{1}\left(M_{n}\right) \longrightarrow$ $\mathrm{PSL}_{2}(\mathbf{C})$ be the composition of the homomorphism $\left(f_{n}\right)_{*}: \pi_{1}\left(M_{n}\right) \longrightarrow \pi_{1}\left(N_{n}\right)$ induced from the $f_{n}$ and the holonomy $\operatorname{hol}_{n}: \pi_{1}\left(N_{n}\right) \longrightarrow \mathrm{PSL}_{2}(\mathbf{C})$ of $N_{n}$. Then, this example also implies that $\left\{\rho_{n}\right\}$ is a sequence of torsion-free, discrete representations to $\mathrm{PSL}_{2}(\mathbf{C})$ with $\lim _{n \rightarrow \infty} \operatorname{Vol}\left(\rho_{n}\right)=\lim _{n \rightarrow \infty} \operatorname{Vol}\left(M_{n}\right)<\infty$, but each $\rho_{n}$ is not faithful, where $\operatorname{Vol}\left(\rho_{n}\right)$ is the volume of $\rho_{n}$ in the sense of Goldman [5]. We compare this fact with Goldman's rigidity theorem which says that, for any closed 
hyperbolic 3-manifold $M$, a representation $\rho: \pi_{1}(M) \longrightarrow \operatorname{Isom}^{+}\left(\mathbf{H}^{3}\right)$ is discrete and faithful if and only if $\operatorname{Vol}(\rho)=\operatorname{Vol}(M)$, see Dunfield [2] Theorem 6.1] for a clear proof.

The following corollary is a stronger version of Gromov-Thurston's Rigidity Theorem.

Corollary 1. For any $V>0$, there exists a constant $c=c(V)>0$ depending only on $V$ and satisfying the following.

(0.2) Let $f: M \longrightarrow N$ be a degree-one map between closed, connected, orientable, hyperbolic 3-manifolds with $\operatorname{Vol}(M) \leq V$. Then, $f$ is homotopic to an isometry if and only if $(1-c) \operatorname{Vol}(M) \leq \operatorname{Vol}(N)$.

Note that Theorem 1 implies that $\lim _{V \rightarrow \infty} c(V)=0$ for any $c(V)$ satisfying the property $(0.2)$. From this rigidity theorem, we know that there are no degree-one maps between distinct 3-manifolds obtained by most Dehn surgeries on the same hyperbolic knot in $S^{3}$. Corollary 2 is another application of Corollary 1, which concerns one of the main subjects on degree-one maps between 3-manifolds, for example see [10, Problem 3.100], [18, [19] and [15].

Corollary 2. For any $V>0$, there exists a natural number $n_{0}=n_{0}(V)$ depending only on $V$ and satisfying the following.

(0.3) Consider any ascending sequence

$$
M_{0} \stackrel{f_{1}}{\longleftarrow} M_{1} \stackrel{f_{2}}{\longleftarrow} M_{2} \stackrel{f_{3}}{\longleftarrow} \cdots \stackrel{f_{n}}{\longleftarrow} M_{n}
$$

of non-homotopy equivalence, degree-one maps between closed, connected, orientable, hyperbolic 3-manifolds. If $n \geq n_{0}$, then the volume of $M_{n}$ is greater than $V$.

Since the set $\mathbf{V}$ of volumes of closed hyperbolic 3-manifolds is a well ordered subset of $\mathbf{R}$ (see [20, Corollary 6.6.1]), any strictly decreasing sequence in $\mathbf{V}$ is finite. From this fact, we know that any descending sequence of non-homotopy equivalence, degree-one maps between closed hyperbolic 3-manifolds is finite. On the other hand, strictly increasing, infinite sequences in $\mathbf{V}$ are not necessarily unbounded. However, Corollary 2 implies that such a sequence in $\mathbf{V}$ defined from ascending sequence of degree-one maps is always unbounded.

We will finish this section by outlining how to prove our rigidity theorem on degree-one maps $f: M \longrightarrow N$ between closed, hyperbolic 3-manifolds with $\operatorname{Vol}(M) / \operatorname{Vol}(N)$ sufficiently close to 1 . As will be seen in Lemmas 1 and 2 in $\S 1$, for "most" of the straight 3-simplices $\sigma: \Delta^{3} \longrightarrow M$ in $M$ well approximated by regular ideal simplices, the straightened 3-simplex straight $(f \circ \sigma)$ of $f \circ \sigma$ in $N$ is also approximated by such an ideal simplex. By using this fact, we wish to show that a lift $\widetilde{f}: \mathbf{H}^{3} \longrightarrow \mathbf{H}^{3}$ of $f$ to the universal coverings is close to a Möbius transformation in a domain near the boundary $\partial \mathbf{H}^{3}=\mathbf{C} \cup\{\infty\}$. Lemma 6 implies that there exists a certain subset $W$ of $\mathbf{H}^{3}=\mathbf{C} \times \mathbf{R}_{+}$adjacent to $\partial \mathbf{H}^{3}$ such that $\widetilde{f} \mid W$ is close to the identity. In fact, by using a simplicial honeycomb $\mathcal{H}_{z, t}^{(m)}$ satisfying a certain effectiveness condition, one can show that $\widetilde{f} \mid V_{z}^{(m)} \times\{t\}$ is close to the identify if we normalize $\widetilde{f}$ by composing some isometric transformation on $\mathbf{H}^{3}$ (Lemma 3 ), where $V_{z}^{(m)}$ is a subset of $\mathbf{C}$ consisting of vertices of $9^{m}$ regular subtriangles of the same size in a certain regular triangle $\widehat{T}_{z}$. Then, a simplicial twin $\mathcal{T}_{z, t}$ is used to show that such a normalization of $\widetilde{f}$ is taken independently of parameters $(z, t)$ (Lemma 4). The subset $W$ as above is contained in the union of $V_{z}^{(m)} \times\{t\}$ 's for "most" 
$(z, t)$ in the parameter space $B_{r}(2 R) \times(0, s] \subset \mathbf{C} \times \mathbf{R}_{+}$given in $\S 2$. Then, we will show that $f \mid M_{\text {thick }(\varepsilon)}$ is homotopic to a homeomorphism $j: M_{\operatorname{thick}(\varepsilon)} \longrightarrow N_{\text {thick }(\varepsilon)}$ by relying on the fact that $\widetilde{f} \mid W$ is close to the identity. Since $j$ maps a meridian of each component of $M_{\operatorname{thin}(\varepsilon)}$ to a meridian of the corresponding component of $N_{\text {thin }(\varepsilon)}, j$ is extended to a homeomorphism $\widehat{j}: M \longrightarrow N$, which is homotopic to an isometry.

Acknowledgement. The author would like to thank the referee for his/her valuable comments and suggestions which were helpful to improve many parts of the original paper.

\section{Preliminaries}

Throughout the paper, all manifolds are assumed to be orientable, and suitably oriented in all cases. First of all, we will review briefly the fundamental notation and definitions needed in later sections, and refer to Thurston 20 for details on hyperbolic geometry, and to Hempel [8, Jaco 9] for 3-manifold topology.

Let $\mathbf{C} \times \mathbf{R}_{+}=\{(z, t) \in \mathbf{C} \times \mathbf{R} ; t>0\}$ be the 3-dimensional upper half space with the Euclidean metric $d s_{E}^{2}=|d z|^{2}+d t^{2}$. Hyperbolic 3-space $\mathbf{H}^{3}$ is the Riemannian 3-manifold with the underlying space $\mathbf{C} \times \mathbf{R}_{+}$and the metric $d s_{H}^{2}=d s_{E}^{2} / t^{2}$. The group $\mathrm{Isom}^{+}\left(\mathbf{H}^{3}\right)$ of orientation-preserving, isometric transformations on $\mathbf{H}^{3}$ is naturally identified with the group $\mathrm{PSL}_{2}(\mathbf{C})$ of Möbius transformations on the Riemann sphere $\widehat{\mathbf{C}}=\mathbf{C} \cup\{\infty\}$. It is easily seen that the compact-open topology on $\operatorname{Isom}^{+}\left(\mathbf{H}^{3}\right)$ coincides with the quotient topology on $\mathrm{PSL}_{2}(\mathbf{C})$ induced from that on $\mathrm{SL}_{2}(\mathbf{C}) \subset \mathbf{C}^{4}$. The quotient space $M=\mathbf{H}^{3} / \Gamma$ of $\mathbf{H}^{3}$ by a torsion-free, discrete subgroup $\Gamma$ of Isom ${ }^{+}\left(\mathbf{H}^{3}\right)$ is called a hyperbolic 3-manifold. Then, the quotient map $p: \mathbf{H}^{3} \longrightarrow M$ is the locally isometric, universal covering. For an $\varepsilon>0$, the $\varepsilon$-thin part $M_{\operatorname{thin}(\varepsilon)}$ of $M$ is the set consisting of all points $x \in M$ such that there exists a non-contractible loop $l$ in $M$ with $l \ni x$ and of length $\leq \varepsilon$. The complement $M_{\text {thick }(\varepsilon)}=M-\operatorname{int} M_{\operatorname{thin}(\varepsilon)}$ is called the $\varepsilon$-thick part of $M$. A Margulis tube is an embedded, equidistant, tubular neighborhood of a short closed geodesic in $M$. A $\mathbf{Z}$ or a $\mathbf{Z} \times \mathbf{Z}$-cusp $C$ is a subset of $M$ such that each component of $p^{-1}(C)$ is a horoball the stabilizer of which in $\Gamma$ is isomorphic to either $\mathbf{Z}$ or $\mathbf{Z} \times \mathbf{Z}$. By the Margulis Lemma [20, Corollary 5.10.2], there exists an $\varepsilon_{0}>0$ independent of $\Gamma$ such that, for any $\varepsilon>0$ less than $\varepsilon_{0}$, each component of $M_{\text {thick }(\varepsilon)}$ is either a Margulis tube or $\mathbf{Z}$ or $\mathbf{Z} \times \mathbf{Z}$-cusp. The $M$ is called geometrically finite if $M$ contains a convex core $C(M)$ such that $C(M) \cap M_{\text {thick }(\varepsilon)}$ is compact. If $\operatorname{Vol}(M)<\infty$, then $M$ has at most finitely many Margulis tubes. If necessary replacing $\varepsilon$ by a sufficiently smaller positive number, we may assume that $M_{\operatorname{thin}(\varepsilon)}$ has no Margulis tube components. Then, each component of $M_{\operatorname{thin}(\varepsilon)}$ is a $\mathbf{Z} \times \mathbf{Z}$-cusp.

Let $\Delta^{k}$ be a regular $k$-simplex of edge length 1 in the Euclidean $k$-space. For any smooth manifold $N$, let $C^{1}\left(\Delta^{k}, N\right)$ be the topological space of $C^{1}$-maps $\Delta^{k} \longrightarrow N$ with $C^{1}$-topology. We denote by $\mathcal{C}_{k}(N)$ the $\mathbf{R}$-vector space consisting of Borel measures $\mu$ on $C^{1}\left(\Delta^{k}, N\right)$ with compact support and bounded total variation. Here, the total variation $\|\mu\|$ of $\mu$ is defined by

$$
\|\mu\|=\int_{C^{1}\left(\Delta^{k}, N\right)} d \mu_{+}+\int_{C^{1}\left(\Delta^{k}, N\right)} d \mu_{-}
$$

where $\mu_{+}, \mu_{-}$are the positive and negative parts of $\mu$ respectively, that is, $\mu_{+} \geq$ $0, \mu_{-} \geq 0$ and $\mu=\mu_{+}-\mu_{-}$. An element of $\mathcal{C}_{k}(N)$ is called a $k$-chain. The boundary 
operator $\partial_{k}: \mathcal{C}_{k}(N) \longrightarrow \mathcal{C}_{k-1}(N)$ is naturally defined. Then, the $\mathbf{R}$-vector space of $k$-cycles is denoted by $\mathcal{Z}_{k}(N)$. The homology determined by $\left(\mathcal{C}_{*}(N), \partial_{*}\right)$ is equal to the standard homology $H_{*}(N, \mathbf{R})$ for $N$, for example see Zastrow [25]. Here, the duality between the homology and cohomology is given by

$$
\langle\mu, \omega\rangle=\int_{C^{1}\left(\Delta^{k}, N\right)}\left(\int_{\Delta^{k}} \sigma^{*}(\omega)\right) d \mu(\sigma)
$$

where $\mu \in \mathcal{Z}_{k}(N)$ and $\omega$ is a closed $k$-form on $N$, see [20, Chapter 6] for details.

Here, we return to the case where $M$ is a hyperbolic 3-manifold with the universal covering $p: \mathbf{H}^{3} \longrightarrow M$. For any continuous map $\widetilde{\sigma}: \Delta^{3} \longrightarrow \mathbf{H}^{3}$, let $\operatorname{straight}(\widetilde{\sigma})$ : $\Delta^{3} \longrightarrow \mathbf{H}^{3}$ be the affine map with respect to the Euclidean structure on $\Delta^{3}$ and the quadratic model on $\mathbf{H}^{3}$ such that straight $\left(\widetilde{\sigma}\left(v_{j}\right)\right)=\widetilde{\sigma}\left(v_{j}\right)$ for all vertices $v_{1}, \ldots, v_{4}$ of $\Delta^{3}$. We note that the image straight $(\widetilde{\sigma})\left(\Delta^{3}\right)$ is a (possibly degenerate) straight 3 -simplex in $\mathbf{H}^{3}$. For a continuous map $\sigma: \Delta^{3} \longrightarrow M$, straight $(\sigma)=p$ ostraight $(\tilde{\sigma})$ : $\Delta^{3} \longrightarrow M$ is called the 3 -simplex obtained by straightening $\sigma$, where $\widetilde{\sigma}: \Delta^{3} \longrightarrow \mathbf{H}^{3}$ is a lift of $\sigma$ to $\mathbf{H}^{3}$. If $z=\sum r_{i} \sigma_{i}\left(r_{i} \in \mathbf{R}\right)$ is a 3 -cycle on $M$, then $\operatorname{straight}(z)=$ $\sum r_{i} \operatorname{straight}\left(\sigma_{i}\right)$ is also a 3 -cycle on $M$ with $[\operatorname{straight}(z)]=[z]$ in $H_{3}(M, \mathbf{R})$.

Now, we suppose that the $M$ is closed. For any non-degenerate, straight 3simplex $\sigma: \Delta^{3} \longrightarrow \mathbf{H}^{3}$, we will define a (Borel measure) 3-cycle $z_{M}(\sigma) \in \mathcal{Z}_{3}(M)$ associated to $\sigma$ such that $\left\|z_{M}(\sigma)\right\|=\operatorname{Vol}(M)$ and $\left[z_{M}(\sigma)\right]=\operatorname{Vol}(\sigma)[M]$ in $H_{3}(M ; \mathbf{R})$, where $[M]$ is the fundamental class of $M$. The 3 -chain $\operatorname{smear}_{M}(\sigma) \in \mathcal{C}_{3}(M)$ satisfying the following (1.2) and (1.3) is defined in [20, Chapter 6] by using a left-right invariant Haar measure $\mu_{0}$ on $\mathrm{Isom}^{+}\left(\mathbf{H}^{3}\right)=\mathrm{PSL}_{2}(\mathbf{C})$. The measure $\mu_{0}$ is normalized so that for any bounded Borel subset $U$ of $\mathbf{H}^{3}$,

$$
\mu_{0}\left(\left\{\alpha \in \operatorname{Isom}^{+}\left(\mathbf{H}^{3}\right) ; \alpha(0,1) \in U\right\}\right)=\operatorname{Vol}(U),
$$

where $(0,1) \in \mathbf{H}^{3}=\mathbf{C} \times \mathbf{R}_{+}$is the base point of $\mathbf{H}^{3}$. The $\mu_{0}$ will play an important role in the proof of the key lemma, Lemma 6 , for Theorem 2.

(1.2) The support $\operatorname{supp}\left(\operatorname{smear}_{M}(\sigma)\right)$ consists of all $C^{1}$-maps $\tau: \Delta^{3} \longrightarrow M$ admitting lifts $\widetilde{\tau}: \Delta^{3} \longrightarrow \mathbf{H}^{3}$ with $\widetilde{\tau}=\alpha \circ \sigma$ for some $\alpha \in \operatorname{Isom}^{+}\left(\mathbf{H}^{3}\right)$.

(1.3) Consider any Borel subset $\mathcal{A}$ of $\operatorname{Isom}^{+}\left(\mathbf{H}^{3}\right)$ such that $\mathcal{A} \cap \gamma \mathcal{A}=\emptyset$ for any nontrivial covering transformations $\gamma \in \pi_{1}(M)-\{1\}$. Then, we have $\operatorname{smear}_{M}(\sigma)(A)$ $=\mu_{0}(\mathcal{A})$ for the subset $A=\{p \circ \alpha \circ \sigma ; \alpha \in \mathcal{A}\} \subset \operatorname{supp}\left(\operatorname{smear}_{M}(\sigma)\right)$.

Intuitively, the measure $\operatorname{smear}_{M}(\sigma)$ "smears $M$ evenly (1.3)" "with straight, singular 3-simplices in $M$ isometric to $\sigma(1.2)$ ". Then, we define $z_{M}(\sigma) \in \mathcal{Z}_{3}(M)$ by

$$
z_{M}(\sigma)=\frac{1}{2}\left(\operatorname{smear}_{M}(\sigma)-\operatorname{smear}_{M}\left(\sigma_{-}\right)\right),
$$

where $\sigma_{-}: \Delta^{3} \longrightarrow \mathbf{H}^{3}$ is a mirror image of $\sigma$. Since, by (1.3) and the normalization of $\mu_{0},\left\|\operatorname{smear}_{M}(\sigma)\right\|=\left\|\operatorname{smear}_{M}\left(\sigma_{-}\right)\right\|=\operatorname{Vol}(M),\left\|z_{M}(\sigma)\right\|$ is also equal to $\operatorname{Vol}(M)$. Moreover, by (1.1) and (1.2),

$$
\left\langle z_{M}(\sigma), \Omega_{M}\right\rangle=\int_{C^{1}\left(\Delta^{3}, M\right)}\left(\int_{\Delta^{3}} \tau^{*}\left(\Omega_{M}\right)\right) d\left(z_{M}(\sigma)\right)(\tau)=\operatorname{Vol}(\sigma) \operatorname{Vol}(M),
$$

where $\Omega_{M}$ is the volume form on $M$. This implies that $\left[z_{M}(\sigma)\right]=\operatorname{Vol}(\sigma)[M]$ in $H_{3}(M, \mathbf{R})$. Even in the case where $M$ is of finite volume but not closed, $z_{M}(\sigma)$ is defined similarly. Then, $z_{M}(\sigma)$ is a measure on $C^{1}\left(\Delta^{3}, M\right)$ with locally compact support, and represents the element $\operatorname{Vol}(\sigma)[M]$ in the locally finite homology $H_{3}^{\text {l.f. }}(M, \mathbf{R})$. 
Let $\left\{N_{n}\right\}$ be a sequence of Riemannian manifolds (possibly with boundary) of the same dimension such that each $N_{n}$ has a base point $x_{n}$. We say that $\left\{N_{n}\right\}$ converges smoothly to a Riemannian manifold $N$ with a base point $x_{0}$ if there exist sequences $\left\{\varepsilon_{n}\right\},\left\{R_{n}\right\}$ with $\varepsilon_{n} \searrow 0, R_{n} \nearrow \infty$ and diffeomorphisms $f_{n}: \mathcal{N}_{R_{n}}\left(x_{n}, N_{n}\right) \longrightarrow$ $\mathcal{N}_{R_{n}}\left(x_{0}, N\right)$ satisfying $1-\varepsilon_{n}<\left\|D f_{n}(v)\right\|<1+\varepsilon_{n}$ for all unit tangent vectors $v \in T_{x}\left(N_{n}\right)$ with $x \in \mathcal{N}_{R_{n}}\left(x_{n}, N_{n}\right)$. Here, $\mathcal{N}_{R}(x, N)$ denotes the $R$-neighborhood of $x$ in $N$. When $n \in \mathbf{N}$ is sufficiently large, $N_{n}$ is said to be well approximated by $N$. Let $\left\{\Delta_{n}\right\}_{n=1}^{\infty}$ be a set of non-degenerate, straight 3-simplices in $\mathbf{H}^{3}$ such that the base point of each $\Delta_{n}$ is its internal center. According to 20, Chapter $7],\left\{\Delta_{n}\right\}$ converges smoothly to a regular ideal 3-simplex $\Delta_{\text {reg }}$ in $\mathbf{H}^{3}$ if and only if $\lim _{n \rightarrow \infty} \operatorname{Vol}\left(\Delta_{n}\right)=\operatorname{Vol}\left(\Delta_{\text {reg }}\right)$. Throughout the remainder of this paper, we set $\mathbf{v}_{3}=\operatorname{Vol}\left(\Delta_{\text {reg }}\right)$.

Let $X, N$ be hyperbolic 3-manifolds of finite volume, and $p: \mathbf{H}^{3}=\widetilde{X} \longrightarrow X$ the universal covering of $X$. A continuous map $\varphi: X \longrightarrow N$ can be lifted to a map $\widetilde{\varphi}: \widetilde{X} \longrightarrow \widetilde{N}$ between the universal coverings. For any $\eta>0$, a non-degenerate, straight 3-simplex $\sigma: \Delta^{3} \longrightarrow \widetilde{X}$ is said to be $\eta$-effective (resp. $\eta$-ineffective) with respect to $\widetilde{\varphi}$ if $\iota \operatorname{Vol}(\operatorname{straight}(\widetilde{\varphi} \circ \sigma))>\mathbf{v}_{3}-\eta\left(\operatorname{resp} . \iota \operatorname{Vol}(\operatorname{straight}(\widetilde{\varphi} \circ \sigma)) \leq \mathbf{v}_{3}-\eta\right)$, where $\iota=\operatorname{Vol}(\sigma) /|\operatorname{Vol}(\sigma)|$. Since $\widetilde{\varphi}$ is continuous, the $\eta$-effectiveness is an open condition. That is, any straight 3 -simplex $\tau: \Delta^{3} \longrightarrow X$ close to an $\eta$-effective 3 -simplex $\sigma$ in $C^{1}\left(\Delta^{3}, X\right)$ is also $\eta$-effective. In particular, there exists an open neighborhood $\mathcal{U}$ of the identity in $\operatorname{Isom}^{+}\left(\mathbf{H}^{3}\right)$ such that $\alpha \circ \sigma$ is $\eta$-effective for any $\alpha \in \mathcal{U}$. We say that a straight 3-simplex $\tau: \Delta^{3} \longrightarrow X$ is $\eta$-effective with respect to $\varphi$ if its lift $\widetilde{\tau}: \Delta^{3} \longrightarrow \widetilde{X}$ is $\eta$-effective with respect to $\widetilde{\varphi}$. For a straight 3 -simplex $\sigma: \Delta^{3} \longrightarrow \mathbf{H}^{3}$, let $\mathcal{C}_{\text {effec }}^{\eta}$ be the subset of $\operatorname{supp}\left(z_{X}(\sigma)\right)$ consisting of $\eta$-effective 3 -simplices with respect to $\varphi$, and set $\mathcal{C}_{\text {ineffec }}^{\eta}=\operatorname{supp}\left(z_{X}(\sigma)\right)-\mathcal{C}_{\text {effec }}^{\eta}$. Then, we have

$$
z_{X}(\sigma)=z_{\text {effec }}^{\eta}+z_{\text {ineffec }}^{\eta} \text { and } \quad\left\|z_{X}(\sigma)\right\|=\operatorname{Vol}(X)=\left\|z_{\text {effec }}^{\eta}\right\|+\left\|z_{\text {ineffec }}^{\eta}\right\|
$$

where $z_{\text {effec }}^{\eta}=z_{X}(\sigma) \mid \mathcal{C}_{\text {effec }}^{\eta}$ and $z_{\text {ineffec }}^{\eta}=z_{X}(\sigma) \mid \mathcal{C}_{\text {ineffec }}^{\eta}$.

Here, let us suppose that (i) $X_{\operatorname{thin}(\delta)}$ consists only of $\mathbf{Z} \times \mathbf{Z}$-cusps for some $\delta>0$, (ii) $N$ is closed, and (iii) $\varphi(P)$ is either a closed geodesic or a point in $N$ for each component $P$ of $X_{\operatorname{thin}(\delta)}$. In the former case of the condition (iii), we assume moreover that $\widetilde{\varphi}(\widetilde{P})$ is a geodesic line in $\widetilde{N}$ for any component $\widetilde{P}$ of $p^{-1}(P)$. The last condition (iii) is used to show that, for any 3 -cycle $z$ in $\left(X, X_{\operatorname{thin}(\delta)}\right)$, $\operatorname{straight}\left(\varphi_{*}(z)\right)$ is a 3 -cycle in $(N, \varphi(X, \operatorname{thin}(\delta)))$. In fact, for any singular 2-simplex $\tau: \Delta^{2} \longrightarrow X_{\operatorname{thin}(\delta)}$, a lift $\widetilde{\varphi \circ \tau}: \Delta^{2} \longrightarrow \mathbf{H}^{3}$ of $\varphi \circ \tau$ is contained in a geodesic (or a point) $\widetilde{\varphi}(\widetilde{P})$, so straight $(\widetilde{\varphi \circ \tau})$ is also in $\widetilde{\varphi}(\widetilde{P})$. Thus, $\partial \operatorname{straight}\left(\varphi_{*}(z)\right)=$ straight $\left(\varphi_{*}(\partial z)\right)$ is a 2 -cycle in $\varphi\left(X_{\operatorname{thin}(\delta)}\right)$. The fundamental classes of $\left(X, X_{\operatorname{thin}(\delta)}\right)$ and $\left(N, \varphi\left(X_{\operatorname{thin}(\delta)}\right)\right)$ are denoted respectively by $\left[X, X_{\operatorname{thin}(\delta)}\right] \in H_{3}\left(X, X_{\operatorname{thin}(\delta)} ; \mathbf{Z}\right) \cong$ $\mathbf{Z}$ and $\left[N, \varphi\left(X_{\operatorname{thin}(\delta)}\right)\right] \in H_{3}\left(N, \varphi\left(X_{\operatorname{thin}(\delta)}\right) ; \mathbf{Z}\right) \cong H_{3}(N ; \mathbf{Z}) \cong \mathbf{Z}$. Then, we have the following lemma.

Lemma 1. If $\varphi_{*}\left(\left[X, X_{\operatorname{thin}(\delta)}\right]\right)=\left[N, \varphi\left(X_{\operatorname{thin}(\delta)}\right)\right]$ and $\operatorname{Vol}(\sigma)>\mathbf{v}_{3}-\varepsilon$ for some $\varepsilon>0$, then

$$
\left\|z_{\text {ineffec }}^{\eta}\right\|<\frac{\mathbf{v}_{3}}{\eta}(\operatorname{Vol}(X)-\operatorname{Vol}(N))+\frac{\varepsilon}{\eta} \operatorname{Vol}(N) .
$$


Proof. Since $\left[z_{X}(\sigma)\right]=\operatorname{Vol}(\sigma)[X]$ in $H_{3}^{\text {l.f. }}(X ; \mathbf{R}), \varphi_{*}\left(z_{X}(\sigma)\right)$ represents $\operatorname{Vol}(\sigma) \times$ $\left[N, \varphi\left(X_{\operatorname{thin}(\delta)}\right)\right]$ in $H_{3}\left(N, \varphi\left(X_{\operatorname{thin}(\delta)}\right) ; \mathbf{R}\right)$. This shows that

$$
\begin{aligned}
\operatorname{Vol}(\sigma) \operatorname{Vol}(N) & =\left\langle\varphi_{*}\left(z_{X}(\sigma)\right), \Omega_{N}\right\rangle \\
& =\left\langle\operatorname{straight}\left(\varphi_{*}\left(z_{X}(\sigma)\right)\right), \Omega_{N}\right\rangle \\
& =\left\langle\operatorname{straight}\left(\varphi_{*}\left(z_{\text {effec }}^{\eta}\right)\right), \Omega_{N}\right\rangle+\left\langle\operatorname{straight}\left(\varphi_{*}\left(z_{\text {ineffec }}^{\eta}\right)\right), \Omega_{N}\right\rangle \\
& \leq \mathbf{v}_{3}\left\|z_{\text {effec }}^{\eta}\right\|+\left(\mathbf{v}_{3}-\eta\right)\left\|z_{\text {ineffec }}^{\eta}\right\|,
\end{aligned}
$$

where $\Omega_{N}$ is the volume form on $N$. Thus, our desired inequality is obtained from the facts $\left\|z_{\text {effec }}^{\eta}\right\|=\operatorname{Vol}(X)-\left\|z_{\text {ineffec }}^{\eta}\right\|$ and $\operatorname{Vol}(\sigma)>\mathbf{v}_{3}-\varepsilon$.

Let $\mathcal{F}$ be a measurable subset of $\operatorname{Isom}^{+}\left(\mathbf{H}^{3}\right)$ such that $\mathcal{F} \cap \gamma \mathcal{F}=\emptyset$ for any nontrivial covering transformation $\gamma \in \pi_{1}(X)-\{1\}$ of $\mathbf{H}^{3}$ and such that $\alpha \circ \sigma: \Delta^{3} \longrightarrow$ $\mathbf{H}^{3}$ is $\eta$-ineffective with respect to $\widetilde{\varphi}$ for any $\alpha \in \mathcal{F}$. Since the correspondence $\alpha \longmapsto p \circ \alpha \circ \sigma$ maps $\mathcal{F}$ injectively into $\mathcal{C}_{\text {ineffec }}^{\eta}$, by $(1.3), \mu_{0}(\mathcal{F}) \leq \operatorname{smear}_{X}(\sigma)\left(\mathcal{C}_{\text {ineffec }}^{\eta}\right)$. Since $2\left\|z_{\text {ineffec }}^{\eta}\right\|=\operatorname{smear}_{X}(\sigma)\left(\mathcal{C}_{\text {ineffec }}^{\eta}\right)+\operatorname{smear}_{X}\left(\sigma_{-}\right)\left(\mathcal{C}_{\text {ineffec }}^{\eta}\right)$, Lemma 1 implies the following.

Lemma 2. With the same assumptions as in Lemma 1,

$$
\mu_{0}(\mathcal{F})<\frac{2 \mathbf{v}_{3}}{\eta}(\operatorname{Vol}(X)-\operatorname{Vol}(N))+\frac{2 \varepsilon}{\eta} \operatorname{Vol}(N) .
$$

Lemma 2 means that, for any subset $\mathcal{F}$ as above, $\mu_{0}(\mathcal{F})$ is small enough if both $\varepsilon>0$ and $\operatorname{Vol}(X)-\operatorname{Vol}(N)$ are sufficiently small compared with $\eta$.

\section{Simplicial honeycombs AND TWins}

For an $a>0$ and $z \in \mathbf{C}$, we denote by $B_{a}(z)$ the disk in $\mathbf{C}$ centered at $z$ of radius $a$. Let $R, r, s$ be positive numbers with $r<R$ and $s<1$. For a $z \in B_{r}(2 R)$, consider the regular triangle $\widehat{T}_{z}$ in $\mathbf{C}$ centered at the origin $0 \in \mathbf{C}$ and such that $z$ is a vertex of $\widehat{T}_{z}$. Divide $\widehat{T}_{z}$ into $9^{m}$ regular triangles $T_{z, 1}, T_{z, 2}, \ldots, T_{z, 9^{m}}$ of the same size for $m \in \mathbf{N}$. These subtriangles are numbered so that the first six triangles

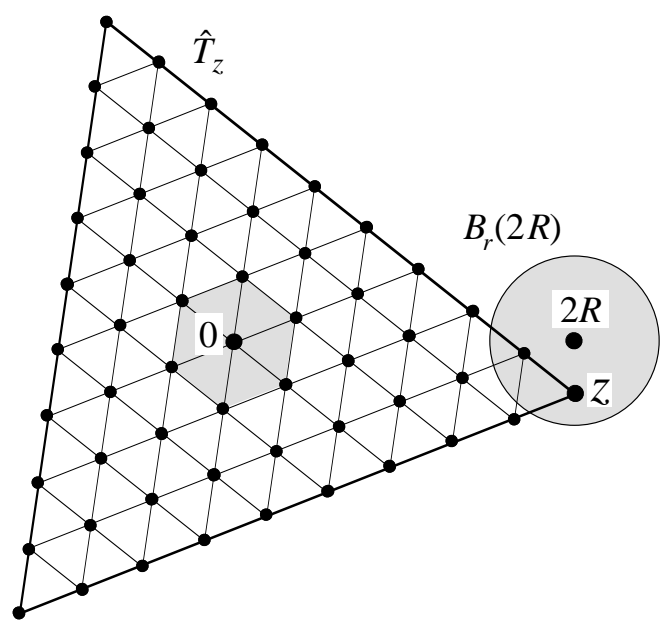

Figure 1 . The case of $m=2$. The shaded hexagon represents the union $T_{z, 1} \cup \cdots \cup T_{z, 6}$. 
$T_{z, 1}, \ldots, T_{z, 6}$ have the origin of $\mathbf{C}$ as a common vertex, see Fig. 1. Let $\left\{v_{i 0}, v_{i 1}, v_{i 2}\right\}$ be the set of vertices of $T_{z, i}$, and let $v_{i 0}=0$ if $i=1,2, \ldots, 6$. Note that the vertex union $V_{z}^{(m)}=\bigcup_{i=1}^{9^{m}}\left\{v_{i 0}, v_{i 1}, v_{i 2}\right\}-\{0\}$ consists of $3^{m+1}\left(3^{m-1}+1\right) / 2$ points evenly distributed in $\widehat{T}_{z}-\{0\}$. For any $i=1,2, \ldots, 6$ and $t \in(0, s]$, consider the straight 3 -simplex $\Delta_{z, i, t}$ in $\mathbf{H}^{3}$ spanned by the four points $(0,1 / s),(0, s),\left(v_{i 1}, t\right)$ and $\left(v_{i 2}, t\right) \in \mathbf{C} \times \mathbf{R}_{+}=\mathbf{H}^{3}$. For $i=7,8, \ldots, 9^{m}$, let $\Delta_{z, i, t}$ be the straight 3 -simplex spanned by $(0,1 / s),\left(v_{i 0}, t\right),\left(v_{i 1}, t\right)$ and $\left(v_{i 2}, t\right)$. We say that the set $\mathcal{H}_{z, t}^{(m)}=\left\{\Delta_{z, i, t} ; i=1,2, \ldots, 9^{m}\right\}$ is the simplicial honeycomb in $\mathbf{H}^{3}$ of type $(z, m, t)$ (for short $(z, m, t)$-honeycomb), see Fig. 2.

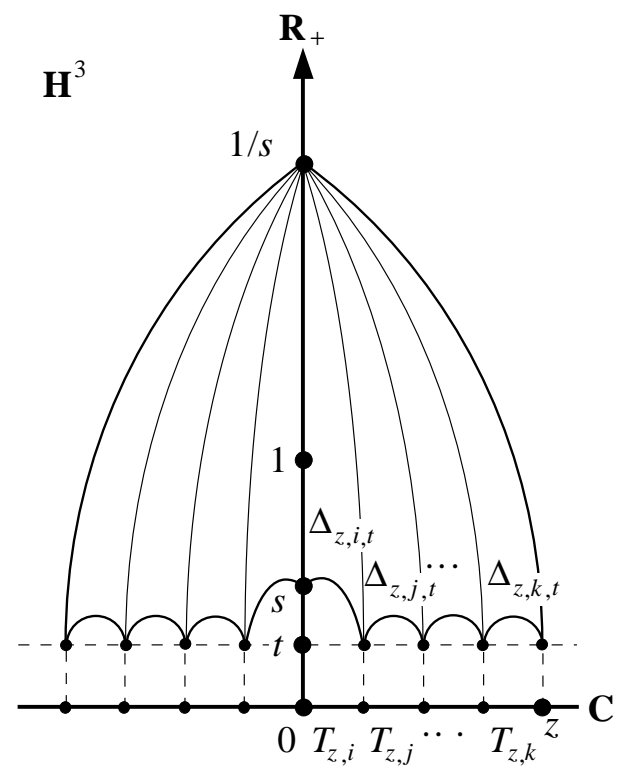

FiguRE 2.

Consider an open condition $\mathbf{P}$ for straight 3 -simplices in $\mathbf{H}^{3}$. Fix an open neighborhood $\mathcal{A}$ of the identity in $\operatorname{Isom}^{+}\left(\mathbf{H}^{3}\right)$. Let $\mathcal{E}^{(m, s)} \subset \mathcal{A} \times B_{r}(2 R) \times(0, s]$ be the $(\alpha, z, t)$ such that $\alpha \Delta_{z, i, t}$ satisfies $\mathbf{P}$ for all $i=1,2, \ldots, 9^{m}$. The set $\mathcal{E}^{(m, s)}$ can be decomposed as follows. Let $\mathcal{F}_{z, i, t}^{(m)}$ be the closed subset of $\mathcal{A}$ consisting of those $\alpha$ for which $\alpha \Delta_{z, i, t}$ does not satisfy $\mathbf{P}$. Then,

$$
\mathcal{E}^{(m, s)}=\left\{(\alpha, z, t) ;(z, t) \in B_{r}(2 R) \times(0, s], \alpha \in \mathcal{A}-\bigcup_{i=1}^{9^{m}} \mathcal{F}_{z, i, t}^{(m)}\right\} .
$$

Since $\mathbf{P}$ is an open condition, $\mathcal{E}^{(m, s)}$ is open in $\mathcal{A} \times B_{r}(2 R) \times(0, s]$.

Let $S_{0}$ be the regular triangle in $\mathbf{C}$ spanned by $\left(0, w_{1}, w_{2}\right)$, where $w_{1}=2 R e^{\pi / 6} / \sqrt{3}$ and $w_{2}=2 R e^{-\pi / 6} / \sqrt{3}$. Note that $\widehat{T}_{2 R}$ can be divided into nine triangles isometric to $S_{0}$. For any $z \in B_{r}(2 R)$, consider the triangle $S_{z}$ in $\mathbf{C}$ spanned by $\left(w_{1}, w_{2}, z\right)$. The triangle $S_{z}$ is regular if and only if $z=2 R$. Let $\nabla_{0}$ be the straight 3 -simplex in $\mathbf{H}^{3}=\mathbf{C} \times \mathbf{R}_{+}$spanned by $(0,1 / s),(0, s),\left(w_{1}, s\right),\left(w_{2}, s\right)$, and let $\nabla_{z, t}$ be that spanned by $(0,1 / s),\left(w_{1}, s\right),\left(w_{2}, s\right),(z, t)$ for $t \in(0, s]$. We say that $\mathcal{T}_{z, t}=\left\{\nabla_{0}, \nabla_{z, t}\right\}$ is the simplicial twin in $\mathbf{H}^{3}$ of type $(z, t)$ (for short $(z, t)$ twin), see Fig. 3. For an open condition $\mathbf{P}^{\prime}$ for straight 3 -simplices in $\mathbf{H}^{3}$, let 


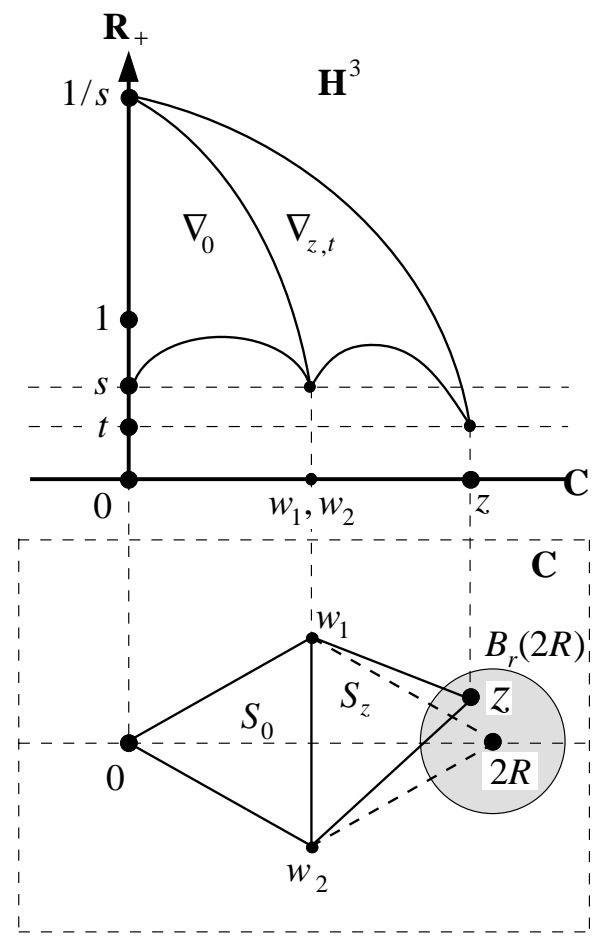

FiguRE 3.

$\mathcal{E}^{\prime} \subset \mathcal{A} \times B_{r}(2 R) \times(0, s]$ be the $(\alpha, z, t)$ such that both $\alpha \nabla_{0}$ and $\alpha \nabla_{z, t}$ satisfy $\mathbf{P}^{\prime}$. The set $\mathcal{E}^{\prime}$ can be decomposed as follows. Let $\mathcal{G}_{0}$ (resp. $\mathcal{G}_{z, t}$ ) be the closed subset of $\mathcal{A}$ consisting of those $\alpha$ for which $\alpha \nabla_{0}$ (resp. $\alpha \nabla_{z, t}$ ) does not satisfy $\mathbf{P}^{\prime}$. Then,

$$
\mathcal{E}^{\prime}=\left\{(\alpha, z, t) ;(z, t) \in B_{r}(2 R) \times(0, s], \alpha \in \mathcal{A}-\mathcal{G}_{0} \cup \mathcal{G}_{z, t}\right\}
$$

Since $\mathbf{P}^{\prime}$ is an open condition, $\mathcal{E}^{\prime}$ is open in $\mathcal{A} \times B_{r}(2 R) \times(0, s]$.

These $\mathcal{E}^{(m, s)}$ and $\mathcal{E}^{\prime}$ for certain open conditions will be used in the proof of Lemma 6. For $\Delta \in \mathcal{H}_{z, t}^{(m)} \cup \mathcal{T}_{z, t}$, any vertex of $\Delta$ other than $(0,1 / s)$ is called a lower vertex. We say that $\mathcal{H}_{z, t}^{(m)}$ (resp. $\mathcal{T}_{z, t}$ ) satisfies $\mathbf{P}$ if each $\Delta$ in $\mathcal{H}_{z, t}^{(m)}$ (resp. in $\mathcal{T}_{z, t}$ ) satisfies $\mathbf{P}$.

For a given continuous map $\widetilde{\varphi}: \mathbf{H}^{3} \longrightarrow \mathbf{H}^{3}$, we will study the $\eta$-effectiveness condition $\mathbf{P}_{\eta}(\widetilde{\varphi})$ with respect to $\widetilde{\varphi}$. That is, a straight 3 -simplex $\Delta$ in $\mathbf{H}^{3}$ of $\operatorname{Vol}(\Delta)>0$ satisfying $\mathbf{P}_{\eta}(\widetilde{\varphi})$ means that $\operatorname{Vol}(\operatorname{straight}(\widetilde{\varphi}(\Delta)))>\mathbf{v}_{3}-\eta$.

Now, let us consider honeycombs and twins under the fixed constant $R$. For any $m \in \mathbf{N}$, there exists a constant $\varepsilon=\varepsilon_{m}(s)>0$ with $\lim _{s \rightarrow 0} \varepsilon_{m}(s)=0$ and such that each $\Delta_{z, i, t}$ in $\mathcal{H}_{z, t}^{(m)}$ has the volume greater than $\mathbf{v}_{3}-\varepsilon$ if $(z, t) \in B_{r}(2 R) \times(0, s]$. For a $(z, t) \in B_{r}(2 R) \times(0, s]$, if necessary deforming $\widetilde{\varphi}$ slightly by homotopy, one can suppose that $\widetilde{\varphi}(0,1 / s), \widetilde{\varphi}(0, s), \widetilde{\varphi}(z, t)$ are not on any single geodesic line in $\mathbf{H}^{3}$. Then, the composition $\widetilde{\varphi}^{(z, t)}=\beta \circ \widetilde{\varphi}: \mathbf{H}^{3} \longrightarrow \mathbf{H}^{3}$ is called the $(z, t)$-normalization of $\varphi$ if we choose $\beta \in \operatorname{Isom}^{+}\left(\mathbf{H}^{3}\right)$ so that $\widetilde{\varphi}^{(z, t)}(0,1 / s)_{\mathbf{C}}=\widetilde{\varphi}^{(z, t)}(0, s)_{\mathbf{C}}=0$, $\widetilde{\varphi}^{(z, t)}(0, s)_{\mathbf{R}}<\widetilde{\varphi}^{(z, t)}(0,1 / s)_{\mathbf{R}}$ and $\widetilde{\varphi}^{(z, t)}(z, t)_{\mathbf{C}}=z$. Here, we set $x_{\mathbf{C}}=z$ and $x_{\mathbf{R}}=t$ for a point $x=(z, t) \in \mathbf{H}^{3}=\mathbf{C} \times \mathbf{R}_{+}$. 


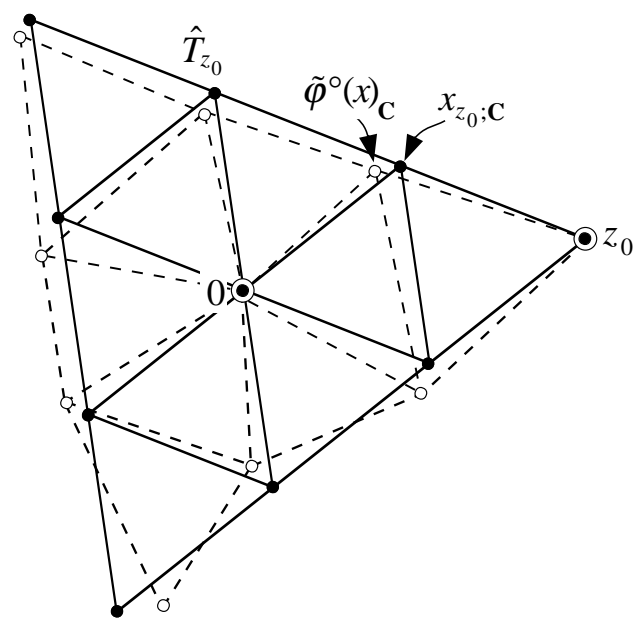

Figure 4. The case of $m=1$. The dots $\bullet$ and $\circ$ represent points of $V_{z_{0}}^{(m)} \cup\{0\}$ and $\widetilde{\varphi}^{\circ}\left(V_{z}^{(m)} \times\{t\}\right)_{\mathbf{C}} \cup\{0\}$ respectively.

Lemma 3. Let us take an arbitrarily small $\delta>0$, and suppose that $r<\delta / 8$. Then, there exist constants $s_{1}=s_{1}(\delta, m)>0$ and $\eta_{1}=\eta_{1}(\delta, m)>0$ such that, if $\mathcal{H}_{z, t}^{(m)}$ satisfies $\mathbf{P}_{\eta_{1}}(\widetilde{\varphi})$ for $(z, t) \in B_{r}(2 R) \times\left(0, s_{1}\right]$ and if we have the normalization $\widetilde{\varphi}^{\circ}=\widetilde{\varphi}^{\left(z^{\prime}, t^{\prime}\right)}$ with $\widetilde{\varphi}^{\circ}(z, t)_{\mathbf{C}} \in B_{\delta / 8}(2 R)$ for some $\left(z^{\prime}, t^{\prime}\right) \in B_{r}(2 R) \times\left(0, s_{1}\right]$, then

$$
\operatorname{dist}_{\mathbf{C} \times \mathbf{R}_{+}}\left(x, \widetilde{\varphi}^{\circ}(x)\right)<\delta
$$

for all lower vertices $x$ of each $\Delta_{z, i, t} \in \mathcal{H}_{z, t}^{(m)}$.

Proof. Since $\mathbf{v}_{3}-\operatorname{Vol}\left(\Delta_{z, i, t}\right)<\varepsilon_{m}(s)$ and $\mathbf{v}_{3}-\operatorname{Vol}\left(\operatorname{straight}\left(\widetilde{\varphi}\left(\Delta_{z, i, t}\right)\right)\right)<\eta_{1}$, the simplices $\Delta_{z, i, t}$ and straight $\left(\widetilde{\varphi}\left(\Delta_{z, i, t}\right)\right)$ are well approximated by regular ideal simplices. Since both $\widetilde{\varphi}^{\circ}(0, s), \widetilde{\varphi}^{\circ}(0,1 / s)$ are in the vertical ray on the origin $0 \in \mathbf{C}$, for the lower vertices $x, x^{\prime}, x^{\prime \prime}$ of $\Delta_{z, i, t}$, the points $\widetilde{\varphi}^{\circ}(x)_{\mathbf{C}}, \widetilde{\varphi}^{\circ}\left(x^{\prime}\right)_{\mathbf{C}}, \widetilde{\varphi}^{\circ}\left(x^{\prime \prime}\right)_{\mathbf{C}}$ span a triangle $T_{i}^{\prime}$ in $\mathbf{C}$ arbitrarily close to a regular triangle by a constant depending only on $\eta_{1}$. More precisely, after rescaling, $T_{i}^{\prime}$ is $k\left(\eta_{1}\right)$-quasi-isometric to the standard regular triangle, where $k(\eta) \geq 1$ is a constant with $\lim _{\eta \rightarrow 0} k(\eta)=1$. Thus, for $z_{0}=\widetilde{\varphi}^{\circ}(z, t)_{\mathbf{C}}$, the tiling pattern with $T_{i}$ 's is well approximated by that with $T_{z_{0}, i}$ 's paving $\widehat{T}_{z_{0}}$. It follows that $\eta_{1}=\eta_{1}(\delta, m)>0$ can be taken so that

$$
\operatorname{dist}_{\mathbf{C}}\left(x_{z_{0} ; \mathbf{C}}, \widetilde{\varphi}^{\circ}(x)_{\mathbf{C}}\right)<\frac{\delta}{4},
$$

where $x_{z_{0} ; \mathbf{C}}$ is the vertex of $V_{z_{0}}^{(m)} \cup\{0\}$ corresponding to the vertex $x_{\mathbf{C}}$ of $V_{z}^{(m)} \cup\{0\}$, see Fig. 4 . Since both $z, z_{0}$ are contained in $B_{\delta / 8}(2 R)$, we have $\operatorname{dist}_{\mathbf{C}}\left(x_{\mathbf{C}}, x_{z_{0} ; \mathbf{C}}\right)<$ $\delta / 4$. Hence, by $(2.1)$,

$$
\operatorname{dist}_{\mathbf{C}}\left(x_{\mathbf{C}}, \widetilde{\varphi}^{\circ}(x)_{\mathbf{C}}\right)<\frac{\delta}{2} .
$$

Moreover, one can take $s_{1}=s_{1}(\delta, m)>0$ and retake $\eta_{1}>0$ if necessary so that, for any lower vertex $x$ of $\Delta_{z, i, t}$, both $x_{\mathbf{R}}$ and $\widetilde{\varphi}^{\circ}(x)_{\mathbf{R}}$ are less than $\delta / 2$. In particular, 
we have

$$
\left|x_{\mathbf{R}}-\widetilde{\varphi}^{\circ}(x)_{\mathbf{R}}\right|<\frac{\delta}{2} .
$$

The inequalities (2.2) and (2.3) imply

$$
\operatorname{dist}_{\mathbf{C} \times \mathbf{R}_{+}}\left(x, \widetilde{\varphi}^{\circ}(x)\right)<\delta .
$$

This completes the proof.

There exists a constant $\varepsilon^{\prime}=\varepsilon^{\prime}(s, r)>0$ with $\lim _{(s, r) \rightarrow(0,0)} \varepsilon^{\prime}=0$ and such that both $\nabla_{0}, \nabla_{z, t}$ in $\mathcal{T}_{z, t}$ have the volumes greater than $\mathbf{v}_{3}-\varepsilon^{\prime}$ if $(z, t) \in B_{r}(2 R) \times(0, s]$.

Lemma 4. For any $\delta>0,0<s<1$ and $0<r<\delta / 16$, there exists a constant $\eta_{2}=\eta_{2}(\delta)>0$ such that, if both $\mathcal{T}_{z, t}$ and $\mathcal{T}_{z^{\prime}, t^{\prime}}$ satisfy $\mathbf{P}_{\eta_{2}}(\widetilde{\varphi})$ for $(z, t),\left(z^{\prime}, t^{\prime}\right) \in$ $B_{r}(2 R) \times(0, s]$, then $\widetilde{\varphi}^{\circ}(z, t)_{\mathbf{C}}$ is contained in $B_{\delta / 8}(2 R)$, where $\widetilde{\varphi}^{\circ}$ is the $\left(z^{\prime}, t^{\prime}\right)$ normalization of $\widetilde{\varphi}$.

Proof. The proof is done by an argument similar to that in Lemma 3 . Since $\mathcal{T}_{t, z}$ and $\mathcal{T}_{z^{\prime}, t^{\prime}}$ contain the same 3 -simplex $\nabla_{0}$, straight $\left(\widetilde{\varphi}\left(\nabla_{z, t}\right)\right)$ and straight $\left(\widetilde{\varphi}\left(\nabla_{z^{\prime}, t^{\prime}}\right)\right)$ have the three common vertices $\widetilde{\varphi}(0,1 / s), \widetilde{\varphi}\left(w_{1}, s\right), \widetilde{\varphi}\left(w_{2}, s\right)$. If one can choose $\eta_{2}=$ $\eta_{2}(\delta)>0$ sufficiently small, then both straight $\left(\widetilde{\varphi}\left(\nabla_{z, t}\right)\right)$ and $\operatorname{straight}\left(\widetilde{\varphi}\left(\nabla_{z^{\prime}, t^{\prime}}\right)\right)$ are well approximated by a common, regular ideal 3 -simplex. Thus, $\eta_{2}>0$ can be taken so that the Euclidean distance between $\widetilde{\varphi}^{\circ}(z, t)$ and $\widetilde{\varphi}^{\circ}\left(z^{\prime}, t^{\prime}\right)$ in $\mathbf{C} \times \mathbf{R}_{+}$is less than $\delta / 16$. Since $\widetilde{\varphi}^{\circ}\left(z^{\prime}, t^{\prime}\right)_{\mathbf{C}}=z^{\prime}$,

$$
\begin{aligned}
\operatorname{dist}_{\mathbf{C}}\left(2 R, \widetilde{\varphi}^{\circ}(z, t)_{\mathbf{C}}\right) & \leq \operatorname{dist}_{\mathbf{C}}\left(2 R, z^{\prime}\right)+\operatorname{dist}_{\mathbf{C}}\left(\widetilde{\varphi}^{\circ}\left(z^{\prime}, t^{\prime}\right)_{\mathbf{C}}, \widetilde{\varphi}^{\circ}(z, t)_{\mathbf{C}}\right) \\
& \leq r+\operatorname{dist}_{\mathbf{C} \times \mathbf{R}_{+}}\left(\widetilde{\varphi}^{\circ}\left(z^{\prime}, t^{\prime}\right), \widetilde{\varphi}^{\circ}(z, t)\right) \\
& <\frac{\delta}{8} .
\end{aligned}
$$

This completes the proof.

Recall that $V_{z}^{(m)}$ is the set of vertices other than the origin $0 \in \mathbf{C}$ of the $9^{m}$ regular triangles $T_{z, 1}, \ldots, T_{z, 9^{m}}$ in $\widehat{T}_{z}$. We denote by $\operatorname{meas}_{\mathbf{C} \times \mathbf{R}_{+}}(\cdot)$ the standard Euclidean measure on $\mathbf{C} \times \mathbf{R}_{+}$.

The following lemma is essentially implied by Sublemma 4.6 in [17], so we only give an outline of the proof.

Lemma 5. Suppose that $\delta, r, s$ are any positive numbers with $\delta<1, r<R$ and $s<1$. Then, there exists $m=m(\delta, r) \in \mathbf{N}$ independent of $s$ and satisfying

$$
\frac{\operatorname{meas}_{\mathbf{C} \times \mathbf{R}_{+}}\left(\widehat{T}_{2 R} \times(0, s] \cap\left(\bigcup_{(z, t) \in L} V_{z}^{(m)} \times\{t\}\right)\right)}{\operatorname{meas} \mathbf{C} \times \mathbf{R}_{+}\left(\widehat{T}_{2 R} \times(0, s]\right)}>1-\delta,
$$

where $L$ is any open subset of $B_{r}(2 R) \times(0, s]$ with

$$
\operatorname{meas}_{\mathbf{C} \times \mathbf{R}_{+}}(L)>\left(1-\frac{\delta}{2}\right) \operatorname{meas} \mathbf{C} \times \mathbf{R}_{+}\left(B_{r}(2 R) \times(0, s]\right) .
$$

Outline of the Proof. From the Fubini Theorem, it suffices to show the existence of such an $m(\delta, r)$ for any level of height $t \in(0, s]$. Let $f: B_{r}(2 R) \times \mathbf{C} \longrightarrow \mathbf{C}$ be a map defined by $f(z, w)=z w / 2 R$. For any $v \in V_{2 R}^{(m)}, f(z, v)$ is an element of $V_{z}^{(m)}$ corresponding to $v$, and $f\left(B_{r}(2 R) \times\{v\}\right)$ is a disk in $\mathbf{C}$ centered at $v$ of radius $r|v| / 2 R$, which is independent of $m$. Thus, we have

$$
\lim _{m \rightarrow \infty} \operatorname{meas}_{\mathbf{C}}\left(\widehat{T}_{2 R} \cap f\left(B_{r}(2 R) \times V_{2 R}^{(m)}\right)\right)=\operatorname{meas}_{\mathbf{C}}\left(\widehat{T}_{2 R}\right) .
$$


Moreover, since $f(\cdot, v): B_{r}(2 R) \longrightarrow \mathbf{C}, v \in V_{2 R}^{(m)}$, is a similar map, for any Borel subset $\lambda$ of $B_{r}(2 R)$ with

$$
\begin{aligned}
\operatorname{meas}_{\mathbf{C}}(\lambda) & =\left(1-\delta^{\prime}\right) \operatorname{meas}_{\mathbf{C}}\left(B_{r}(2 R)\right), \\
\operatorname{meas}_{\mathbf{C}}(f(\lambda \times\{v\})) & =\left(1-\delta^{\prime}\right) \operatorname{meas}_{\mathbf{C}}\left(f\left(B_{r}(2 R) \times\{v\}\right)\right) .
\end{aligned}
$$

From these facts, one can have a constant $m(\delta, r) \in \mathbf{N}$ satisfying the conclusion of Lemma 5.

\section{Proofs of the MAIn THEOREM AND COROLlaries}

For a constant $K \geq 1$, a homeomorphism $f:\left(X, d_{X}\right) \longrightarrow\left(Y, d_{Y}\right)$ between metric spaces is $K$-quasi-isometric if the map satisfies

$$
K^{-1} d_{X}\left(x, x^{\prime}\right) \leq d_{Y}\left(f(x), f\left(x^{\prime}\right)\right) \leq K d_{X}\left(x, x^{\prime}\right)
$$

for any $x, x^{\prime} \in X$. One can suppose that a $K$-quasi-isometry is close to an isometry if and only if $K$ is close to 1.

Our main theorem, Theorem 2 , is proved by reduction to absurdity. So, we suppose that there exist degree-one maps $f_{n}: M_{n} \longrightarrow N_{n}(n=1,2,3, \ldots)$ such that infinitely many $f_{n}$ 's of them are not homotopic to isometries, where $M_{n}, N_{n}$ are closed, connected, hyperbolic 3-manifolds with $\lim _{n \rightarrow \infty} \operatorname{Vol}\left(M_{n}\right)=$ $\lim _{n \rightarrow \infty} \operatorname{Vol}\left(N_{n}\right)<\infty$. By passing to a subsequence if necessary, we may assume that all $f_{n}$ 's are not homotopic to isometries, and hence they are not homotopy equivalences by Mostow's Rigidity Theorem [13]. By Jørgensen's Theorem (see [20, Chapter 6]), we may also assume that there exist connected, hyperbolic 3manifolds $X, Y$ such that each $M_{n}$ (resp. $N_{n}$ ) is obtained by hyperbolic Dehn surgery on $X$ (resp. on $Y$ ). Moreover, we have sequences $\left\{\varepsilon_{n}\right\},\left\{K_{n}\right\}$ with $\varepsilon_{n} \searrow 0$, $K_{n} \searrow 1$ so that there exist $K_{n}$-quasi-isometries $g_{n}: X_{\operatorname{thick}\left(\varepsilon_{n}\right)} \longrightarrow M_{n, \operatorname{thick}\left(\varepsilon_{n}\right)}$ and $h_{n}: N_{n, \operatorname{thick}\left(\varepsilon_{n}\right)} \longrightarrow Y_{\operatorname{thick}\left(\varepsilon_{n}\right)}$. Our assumption on volume limit implies

$$
\operatorname{Vol}(X)=\operatorname{Vol}(Y)=\lim _{n \rightarrow \infty} \operatorname{Vol}\left(N_{n}\right) .
$$

Take an $\varepsilon_{0}>0$ less than the Margulis constant such that each component of $X_{\operatorname{thin}\left(\varepsilon_{0}\right)}$ or $Y_{\operatorname{thin}\left(\varepsilon_{0}\right)}$ is a $\mathbf{Z} \times \mathbf{Z}$-cusp. Let $p: \mathbf{H}^{3}=\widetilde{X} \longrightarrow X$ be the universal covering. Fix a point $x_{0}$ in $X_{\text {thick }\left(\varepsilon_{0}\right)}$, and choose a point $\widetilde{x}_{0}$ in $\widetilde{X}$ with $p\left(\widetilde{x}_{0}\right)=x_{0}$. The coordinate on $\mathbf{H}^{3}$ is rearranged so that $\widetilde{x}_{0}=(0,1)$ in $\mathbf{H}^{3}=\mathbf{C} \times \mathbf{R}_{+}$.

First of all, we will fix a positive number $R$ and a connected, open neighborhood $\mathcal{A}$ of the identity in $\operatorname{Isom}^{+}\left(\mathbf{H}^{3}\right)$, which are not changed throughout the proof. Let $\gamma_{1}, \ldots, \gamma_{k}$ be a set of generators of $\pi_{1}\left(X, x_{0}\right)$ consisting of non-parabolic elements. We choose $R>0$ such that at least one of the two fixed point of each $\gamma_{i}$ is contained in $B_{R / 3}(0)$. If necessary replacing $\gamma_{i}$ by $\gamma_{i}^{-1}$, we may assume that $B_{R / 3}(0)$ contains the attracting fixed point of $\gamma_{i}$. We choose $\mathcal{A}$ such that (i) $\mathcal{A} \cap \gamma \mathcal{A}=\emptyset$ for any $\gamma \in \pi_{1}\left(X, x_{0}\right)-\{1\}$, and (ii) $\operatorname{dist}_{\mathbf{H}^{3}}\left(\widetilde{x}_{0}, \alpha \widetilde{x}_{0}\right)<\varepsilon_{0}$ and $\alpha^{-1}\left(B_{R / 3}(0)\right) \subset B_{R / 2}(0)$ for any $\alpha \in \mathcal{A}$.

Let us denote a unique closed geodesic of a Margulis tube $V$ in $M_{n}$ by $c_{V}$. If necessary deforming $f_{n}$ by homotopy, we may assume that, for each component $V$ of $M_{n, \operatorname{thin}\left(\varepsilon_{n}\right)}, f_{n}(V)$ is a closed geodesic $l$ in $N_{n}$ if $f_{n} \mid c_{V}$ is homotopic to $l$ in $N_{n}$, and $f_{n}(V)$ is a point if $f_{n} \mid c_{V}$ is contractible in $N_{n}$. For a deformation retract $r_{n}: X \longrightarrow X_{\text {thick }\left(\varepsilon_{n}\right)}$, we set $\varphi_{n}=f_{n} \circ g_{n} \circ r_{n}: X \longrightarrow N_{n}$. Let $\widetilde{\varphi}_{n}: \mathbf{H}^{3}=\widetilde{X} \longrightarrow$ $\mathbf{H}^{3}=\widetilde{N}_{n}$ be a lift of $\varphi_{n}$ to the universal covering. Note that $\widetilde{\varphi}_{n} \circ \gamma=\left(\varphi_{n}\right)_{*}(\gamma) \circ \widetilde{\varphi}_{n}$ 
for any $\gamma \in \pi_{1}\left(X, x_{0}\right)$. Here, $\gamma: \mathbf{H}^{3} \longrightarrow \mathbf{H}^{3}$ is a covering transformation such that, for an oriented $\operatorname{arc} \widetilde{c}$ in $\mathbf{H}^{3}$ connecting $\widetilde{x}_{0}$ with $\gamma\left(\widetilde{x}_{0}\right), p \circ \widetilde{c}$ represents $\gamma \in \pi_{1}\left(X, x_{0}\right)$.

The key to the proof of Theorem 2 is the following lemma.

Lemma 6. Let $\delta>0$ be any positive number. Then, we have $n_{0}=n_{0}(\delta) \in \mathbf{N}$ and $0<s_{0}<1$ such that, for any integer $n \geq n_{0}$, there are $\alpha_{n} \in \mathcal{A}$ and a Borel subset $W_{n}$ of $\widehat{T}_{2 R} \times\left(0, s_{0}\right]$ satisfying the following (3.2) and (3.3).

$$
\begin{gathered}
\operatorname{meas}_{\mathbf{C} \times \mathbf{R}_{+}}\left(W_{n}\right)>(1-\delta) \text { meas }_{\mathbf{C} \times \mathbf{R}_{+}}\left(\widehat{T}_{2 R} \times\left(0, s_{0}\right]\right) . \\
\operatorname{dist}_{\mathbf{C} \times \mathbf{R}_{+}}\left(\widetilde{\varphi}_{n}^{\circ}(x), x\right)<\delta \quad \text { for any } x \in W_{n},
\end{gathered}
$$

where $\widetilde{\varphi}_{n}^{\circ}$ is the $\left(z^{\prime}, t^{\prime}\right)$-normalization of $\widetilde{\varphi}_{n} \circ \alpha_{n}$ for some $\left(z^{\prime}, t^{\prime}\right) \in B_{\delta / 16}(2 R) \times$ $\left(0, s_{0}\right]$.

Proof. Take $\eta_{2}=\eta_{2}(\delta)>0$ satisfying Lemma 4. Since $\lim _{(s, r) \rightarrow(0,0)} \varepsilon^{\prime}(s, r)=0$, there exist $0<s_{2}<1$ and $0<r<\delta / 16$ such that $2 \varepsilon^{\prime} \eta_{2}^{-1} \operatorname{Vol}\left(N_{n}\right)<\delta \mu_{0}(\mathcal{A}) / 16$ for any $0<s \leq s_{2}$ and $n \in \mathbf{N}$, where $\mu_{0}$ is the normalized Haar measure on $\operatorname{Isom}_{+}\left(\mathbf{H}^{3}\right)$ given in $\S 1$. By (3.1), we have $n_{1} \in \mathbf{N}$ such that, for any integer $n \geq n_{1}, 2 \mathbf{v}_{3} \eta_{2}^{-1}\left(\operatorname{Vol}(X)-\operatorname{Vol}\left(N_{n}\right)\right)<\delta \mu_{0}(\mathcal{A}) / 16$. Then, by Lemma 2 ,

$$
\mu_{0}\left(\mathcal{G}_{0} \cup \mathcal{G}_{z, t}\right) \leq \mu_{0}\left(\mathcal{G}_{0}\right)+\mu_{0}\left(\mathcal{G}_{z, t}\right)<\frac{\delta \mu_{0}(\mathcal{A})}{4},
$$

where $\mathcal{G}_{0}, \mathcal{G}_{z, t}$ are the closed subsets of $\mathcal{A}$ given in $\S 2$ for $\mathbf{P}=\mathbf{P}_{\eta_{2}}\left(\widetilde{\varphi}_{n}\right)$.

Let $m \in \mathbf{N}$ be the integer given in Lemma 5 for $\delta>0, r>0$ as above, and let $\eta_{1}=\eta_{1}(\delta, m)>0$ be a constant satisfying Lemma 3. Since $\lim _{s \rightarrow 0} \varepsilon_{m}(s)=0$, there exists $0<s_{3} \leq s_{1}(\delta, m)$ such that $2 \varepsilon_{m}(s) \eta_{1}^{-1} \operatorname{Vol}\left(N_{n}\right)<9^{-m} \delta \mu_{0}(\mathcal{A}) / 8$ for any $0<s \leq s_{3}$ and $n \in \mathbf{N}$. There exists $n_{2} \in \mathbf{N}$ such that, for any integer $n \geq n_{2}$, $2 \mathbf{v}_{3} \eta_{1}^{-1}\left(\operatorname{Vol}(X)-\operatorname{Vol}\left(N_{n}\right)\right)<9^{-m} \delta \mu_{0}(\mathcal{A}) / 8$. Again by Lemma 2 , we have

$$
\sum_{i=1}^{9^{m}} \mu_{0}\left(\mathcal{F}_{z, i, t}^{(m)}\right)<\frac{\delta \mu_{0}(\mathcal{A})}{4}
$$

where $\mathcal{F}_{z, i, t}^{(m)}$ is the closed subset of $\mathcal{A}$ given in $\S 2$ for $\mathbf{P}=\mathbf{P}_{\eta_{1}}\left(\widetilde{\varphi}_{n}\right)$. We set $n_{0}=$ $\max \left\{n_{1}, n_{2}\right\}$ and $s_{0}=\min \left\{s_{2}, s_{3}\right\}$. For any integer $n \geq n_{0}$, let $\mathcal{E}^{\left(m, s_{0}\right)}$ and $\mathcal{E}^{\prime}$ be the open subsets of $\mathcal{A} \times B_{r}(2 R) \times\left(0, s_{0}\right]$ given as in $\S 2$. By (3.4) and (3.5),

$$
\frac{\mu_{0} \times \operatorname{meas}_{\mathbf{C} \times \mathbf{R}_{+}}\left(\mathcal{E}^{\left(m, s_{0}\right)} \cap \mathcal{E}^{\prime}\right)}{\mu_{0} \times \operatorname{meas}_{\mathbf{C} \times \mathbf{R}_{+}}\left(\mathcal{A} \times B_{r}(2 R) \times\left(0, s_{0}\right]\right)}>1-\frac{\delta}{2} .
$$

Thus, we have $\alpha_{n} \in \mathcal{A}$ satisfying

$$
\frac{\operatorname{meas}_{\mathbf{C} \times \mathbf{R}_{+}}\left(L_{n}\right)}{\operatorname{meas} \mathbf{C} \times \mathbf{R}_{+}\left(B_{r}(2 R) \times\left(0, s_{0}\right]\right)}>1-\frac{\delta}{2},
$$

where $L_{n} \subset B_{r}(2 R) \times\left(0, s_{0}\right]$ is the $\alpha_{n}$-section of $\mathcal{E}^{\left(m, s_{0}\right)} \cap \mathcal{E}^{\prime}$, that is,

$$
\left\{\alpha_{n}\right\} \times L_{n}=\left(\left\{\alpha_{n}\right\} \times \mathbf{C} \times \mathbf{R}_{+}\right) \cap \mathcal{E}^{\left(m, s_{0}\right)} \cap \mathcal{E}^{\prime} .
$$

By Lemma 5 ,

$$
W_{n}=\widehat{T}_{2 R} \times\left(0, s_{0}\right] \cap\left(\bigcup_{(z, t) \in L_{n}} V_{z}^{(m)} \times\{t\}\right)
$$

is a Borel subset of $\widehat{T}_{2 R} \times\left(0, s_{0}\right]$ satisfying (3.2). Note that, for any $\left(z^{\prime}, t^{\prime}\right) \in L_{n}$, $\mathcal{H}_{z^{\prime}, t^{\prime}}^{(m)}$ and $\mathcal{T}_{z^{\prime}, t^{\prime}}$ satisfy respectively $\mathbf{P}_{\eta_{1}}\left(\widetilde{\varphi}_{n} \circ \alpha_{n}\right)$ and $\mathbf{P}_{\eta_{2}}\left(\widetilde{\varphi}_{n} \circ \alpha_{n}\right)$. Let $\widetilde{\varphi}_{n}^{\circ}=$ 
$\beta_{n} \circ \widetilde{\varphi}_{n} \circ \alpha_{n}$ be the $\left(z^{\prime}, t^{\prime}\right)$-normalization of $\widetilde{\varphi}_{n} \circ \alpha_{n}$ for a fixed $\left(z^{\prime}, t^{\prime}\right) \in L_{n}$. For any $x \in W_{n}$, there exists $(z, t) \in L_{n}$ so that $x$ is a lower vertex of some $\Delta_{z, i, t} \in \mathcal{H}_{z, t}^{(m)}$. By Lemma $4, \widetilde{\varphi}_{n}^{\circ}(z, t)_{\mathbf{C}}$ is contained in $B_{\delta / 8}(2 R)$. Hence, by Lemma 3 , we have (3.3). This completes the proof.

Proof of Theorem 2. For any $n \in \mathbf{N}$, consider the new coordinate on $\widetilde{X}=\mathbf{H}^{3}=\mathbf{C} \times$ $\mathbf{R}_{+}$with $x^{\text {new }}=\alpha_{n}^{-1}\left(x^{\text {old }}\right)$, and so $\left(\alpha_{n} \widetilde{x}_{0}\right)^{\text {new }}=(0,1)$. The covering transformation $\gamma_{n i}$ of $p: \mathbf{H}^{3} \longrightarrow X$ corresponding to $\gamma_{i} \in \pi_{1}(X)(i=1, \ldots, k)$ with respect to the new coordinate is $\alpha_{n}^{-1} \circ \gamma_{i} \circ \alpha_{n}$. Since $\operatorname{dist}_{\mathbf{H}^{3}}\left(\widetilde{x}_{0}, \alpha_{n} \widetilde{x}_{0}\right)<\varepsilon_{0}$, if necessary passing to a subsequence, we may assume that $\left\{\alpha_{n}\right\}$ converges to $\alpha_{0}$ in $\operatorname{Isom}^{+}\left(\mathbf{H}^{3}\right)$. Then, $\left\{\gamma_{n i}\right\}$ converges to $\gamma_{0 i}=\alpha_{0}^{-1} \circ \gamma_{i} \circ \alpha_{0}$. Note that the attracting fixed point of $\gamma_{n i}$ is contained in $B_{R / 2}(0)$. Similarly, the new coordinate on $\widetilde{N}=\mathbf{C} \times \mathbf{R}_{+}$is defined with the isometry $\beta_{n}: \mathbf{H}^{3} \longrightarrow \mathbf{H}^{3}$ so that the covering transformation $\left(\varphi_{n}\right)_{*}\left(\gamma_{i}\right)^{\text {new }}$ corresponding to $\left(\varphi_{n}\right)_{*}\left(\gamma_{i}\right) \in \pi_{1}(N)$ is $\beta_{n} \circ\left(\varphi_{n}\right)_{*}\left(\gamma_{i}\right)^{\text {old }} \circ \beta_{n}^{-1}$. The equality $\left(\varphi_{n}\right)_{*}\left(\gamma_{i}\right)^{\text {old }} \circ \widetilde{\varphi}_{n}=\widetilde{\varphi}_{n} \circ \gamma_{i}$ implies $\left(\varphi_{n}\right)_{*}\left(\gamma_{i}\right)^{\text {new }} \circ \widetilde{\varphi}_{n}^{\circ}=\widetilde{\varphi}_{n}^{\circ} \circ \gamma_{n i}$. For simplicity, we set $\left(\varphi_{n}\right)_{*}\left(\gamma_{i}\right)^{\text {new }}=\left(\varphi_{n}\right)_{*}\left(\gamma_{i}\right)$.

For any $\delta>0$, let $n_{0}(\delta)$ be a positive integer given in Lemma 6 . There exists a sequence $\left\{\delta_{n}\right\}_{n=\nu}^{\infty}$ with $\delta_{n}>0, \lim _{n \rightarrow \infty} \delta_{n}=0$ and $n \geq n_{0}\left(\delta_{n}\right)$, where $\nu=n_{0}(1)$. Here, we will show that there exists a constant $c_{0}>0$ such that, for the Borel subset $O_{n i}=W_{n} \cap \gamma_{n i}^{-1}\left(W_{n}\right)$ of $W_{n}$,

$$
\liminf _{n \rightarrow \infty} \frac{\operatorname{meas}_{\mathbf{C} \times \mathbf{R}_{+}}\left(O_{n i}\right)}{\operatorname{meas}_{\mathbf{C} \times \mathbf{R}_{+}}\left(W_{n}\right)}>c_{0} .
$$

Since $0<\left|d \gamma_{0 i}\left(z_{0}\right) / d z\right|<1$ at the attracting fixed point $z_{0} \in B_{R / 2}(0)$ of $\gamma_{0 i}$, there are real numbers $c_{1}, c_{2}$ with $0<c_{1}<c_{2}<1$ and a small round disk $D$ in $\widehat{T}_{2 R}$ with $\gamma_{0 i}(D) \subset \operatorname{int} D$ and $c_{1}<\left|d \gamma_{0 i}(z) / d z\right|<c_{2}$ for all $z \in D$. Since $\left\{\gamma_{n i}\right\} \rightarrow \gamma_{0 i}$ in $\operatorname{Isom}^{+}\left(\mathbf{H}^{3}\right)$, if we take $s_{0}>0$ in Lemma 6 small enough, then $c_{1}<\left\|d \gamma_{n i}(\mathbf{v})\right\|<c_{2}$ for all sufficiently large $n \in \mathbf{N}$ and for all unit vectors $\mathbf{v}$ tangent to $D \times\left(0, s_{0}\right]$, where $\|\cdot\|$ denotes the norm on the Euclidean half space $\mathbf{C} \times \mathbf{R}_{+}$. Since $c_{2}<1$ and $\gamma_{n i}^{-1}(D) \supset \operatorname{int} D$, one can suppose $\gamma_{n i}^{-1}\left(D \times\left(0, s_{0}\right]\right) \supset D \times\left(0, s_{0}\right]$. We set $U_{n}=D \times\left(0, s_{0}\right] \cap W_{n}$ and $c_{3}=\operatorname{Area}(D) / \operatorname{Area}\left(\widehat{T}_{2 R}\right)$. By $(3.2)$ of Lemma 6 ,

$$
\text { meas } \mathbf{C} \times \mathbf{R}_{+}\left(U_{n}\right) \geq \operatorname{meas} \mathbf{C} \times \mathbf{R}_{+}\left(D \times\left(0, s_{0}\right]\right)-\delta_{n} \operatorname{meas} \mathbf{C} \times \mathbf{R}_{+}\left(\widehat{T}_{2 R} \times\left(0, s_{0}\right]\right),
$$

and

$$
\begin{aligned}
\operatorname{meas}_{\mathbf{C} \times \mathbf{R}_{+}}\left(\gamma_{n i}^{-1}\left(U_{n}\right)\right) \geq & \operatorname{meas}_{\mathbf{C} \times \mathbf{R}_{+}}\left(\gamma_{n i}^{-1}\left(D \times\left(0, s_{0}\right]\right)\right) \\
& -\delta_{n} c_{1}^{-3} \operatorname{meas}_{\mathbf{C} \times \mathbf{R}_{+}}\left(\widehat{T}_{2 R} \times\left(0, s_{0}\right]\right) .
\end{aligned}
$$

These inequalities imply that

$$
\begin{aligned}
\operatorname{meas}_{\mathbf{C} \times \mathbf{R}_{+}}\left(U_{n} \cap \gamma_{n i}^{-1}\left(U_{n}\right)\right) \geq & \operatorname{meas}_{\mathbf{C} \times \mathbf{R}_{+}}\left(D \times\left(0, s_{0}\right]\right) \\
& -\delta_{n}\left(1+c_{1}^{-3}\right) \operatorname{meas}_{\mathbf{C} \times \mathbf{R}_{+}}\left(\widehat{T}_{2 R} \times\left(0, s_{0}\right]\right) .
\end{aligned}
$$

The situation is illustrated in Fig. 5. Since

$$
\frac{\operatorname{meas}_{\mathbf{C} \times \mathbf{R}_{+}}\left(O_{n i}\right)}{\operatorname{meas}_{\mathbf{C} \times \mathbf{R}_{+}}\left(W_{n}\right)} \geq \frac{\operatorname{meas}_{\mathbf{C} \times \mathbf{R}_{+}}\left(U_{n} \cap \gamma_{n i}^{-1}\left(U_{n}\right)\right)}{\operatorname{meas}_{\mathbf{C} \times \mathbf{R}_{+}}\left(\widehat{T}_{2 R} \times\left(0, s_{0}\right]\right)},
$$

the left hand side of (3.6) is greater than $c_{3}-\delta_{0}\left(1+c_{1}^{-3}\right)$ if $0<\delta_{n}<\delta_{0}$ for a fixed small number $\delta_{0}>0$. This shows (3.6). 

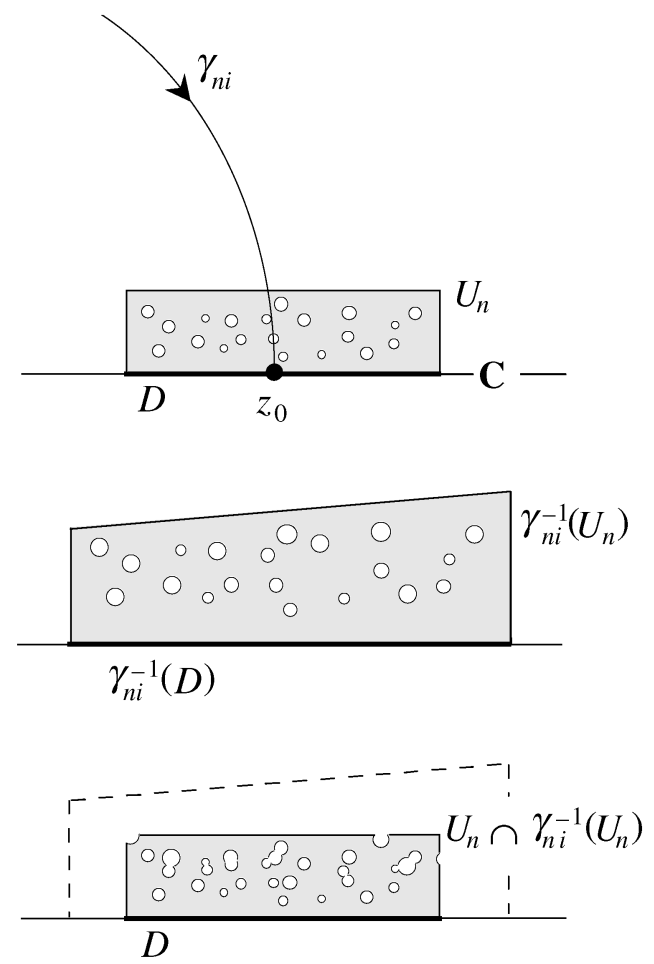

FIGURE 5 .

Let $q_{n}: \mathbf{H}^{3} \longrightarrow N_{n}$ be the universal covering, and let $c_{n i}$ be an oriented loop in $N_{n}$ based at $y_{n}=q_{n}(0,1)$ and representing $\left(\varphi_{n}\right)_{*}\left(\gamma_{i}\right)$. By invoking the two facts that (i) $\left(\varphi_{n}\right)_{*}\left(\gamma_{i}\right) \circ \widetilde{\varphi}_{n}^{\circ}=\widetilde{\varphi}_{n}^{\circ} \circ \gamma_{n i}$ and (ii) $\widetilde{\varphi}_{n}^{\circ}$ satisfies (3.3) in $O_{n i} \cup \gamma_{n i}\left(O_{n i}\right)$, for each $i \in\{1, \ldots, k\}$, we will show the following.

(3.7) The sequence $\left\{\left(\varphi_{n}\right)_{*}\left(\gamma_{i}\right)\right\}$ converges to $\gamma_{0 i}$ in $\operatorname{Isom}^{+}\left(\mathbf{H}^{3}\right)$.

By (3.6), we have a constant $d_{0}>0$ independent of $n$ and three points $a_{n t}$ $(t=1,2,3)$ in $O_{n i}$ satisfying $\operatorname{dist}_{\mathbf{C} \times \mathbf{R}_{+}}\left(a_{n t}, a_{n u}\right), \operatorname{dist}_{\mathbf{C} \times \mathbf{R}_{+}}\left(\gamma_{n i}\left(a_{n t}\right), \gamma_{n i}\left(a_{n u}\right)\right)>d_{0}$ for $1 \leq t, u \leq 3$ with $t \neq u$. By taking a subsequence if necessary, one can

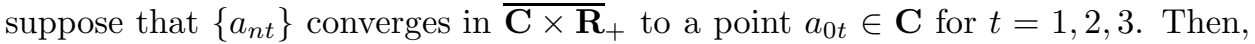
$\left\{\gamma_{n i}\left(a_{n t}\right)\right\}$ converges to $\gamma_{0 i}\left(a_{0 t}\right)$. Since $a_{n t} \in W_{n}$, by (3.3) with $\left\{\delta_{n}\right\} \rightarrow 0$, we have $\operatorname{dist}\left(\widetilde{\varphi}_{n}^{\circ}\left(a_{n t}\right), a_{n t}\right) \rightarrow 0$. Then, the convergence $\left\{\widetilde{\varphi}_{n}^{\circ}\left(a_{n t}\right)\right\} \rightarrow a_{0 t}$ follows from $\operatorname{dist}\left(a_{n t}, a_{0 t}\right) \rightarrow 0$. For the proof of (3.7), we will show the following in advance.

(3.8) $\left\{\left(\varphi_{n}\right)_{*}\left(\gamma_{i}\right) \circ \widetilde{\varphi}_{n}^{\circ}\left(a_{n t}\right)\right\}=\left\{\widetilde{\varphi}_{n}^{\circ} \circ \gamma_{n i}\left(a_{n t}\right)\right\} \rightarrow \gamma_{0 i}\left(a_{0 t}\right)$.

Since $\gamma_{n i}\left(a_{n t}\right) \in W_{n}$, by $(3.3), \operatorname{dist}\left(\widetilde{\varphi}_{n}^{\circ} \circ \gamma_{n i}\left(a_{n t}\right), \gamma_{n i}\left(a_{n t}\right)\right) \rightarrow 0$. Set $K=$ $\widehat{T}_{2 R} \times[0,1] \cap \gamma_{0 i}^{-1}\left(\widehat{T}_{2 R} \times[0,1]\right)$. Since $\gamma_{0 i}$ maps the compact set $K$ into the compact set $\widehat{T}_{2 R} \times[0,1]$ and since $\left\{\gamma_{n i}\right\} \rightarrow \gamma_{0 i}$ in $\operatorname{Isom}^{+}\left(\mathbf{H}^{3}\right),\left\{\gamma_{n i}\right\}$ converges uniformly to $\gamma_{0 i}$ in a small compact neighborhood $N(K)$ of $K$. Since $a_{n 1}, a_{n 2}, a_{n 3} \in N(K)$ for all sufficiently large $n \in \mathbf{N}$, $\operatorname{dist}\left(\gamma_{n i}\left(a_{n t}\right), \gamma_{0 i}\left(a_{n t}\right)\right) \rightarrow 0$. Since $\left\{a_{n t}\right\} \rightarrow a_{0 t}$, $\operatorname{dist}\left(\gamma_{0 i}\left(a_{n t}\right), \gamma_{0 i}\left(a_{0 t}\right)\right) \rightarrow 0$. Thus, we have $\operatorname{dist}\left(\widetilde{\varphi}_{n}^{\circ} \circ \gamma_{n i}\left(a_{n t}\right), \gamma_{0 i}\left(a_{0 t}\right)\right) \rightarrow 0$. This shows (3.8).

By (3.8), $\left\{\gamma_{0 i}^{-1} \circ\left(\varphi_{n}\right)_{*}\left(\gamma_{i}\right) \circ \widetilde{\varphi}_{n}^{\circ}\left(a_{n t}\right)\right\} \rightarrow a_{0 t}$. For simplicity, we set $\psi_{n}=\gamma_{0 i}^{-1} \circ$ $\left(\varphi_{n}\right)_{*}\left(\gamma_{i}\right)$. We have a sequence $\left\{\varepsilon_{n}\right\}$ with $\varepsilon_{n}>0$ and $\lim _{n \rightarrow \infty} \varepsilon_{n}=0$ such that both 
$\widetilde{\varphi}_{n}^{\circ}\left(a_{n t}\right)$ and $\psi_{n} \circ \widetilde{\varphi}_{n}^{\circ}\left(a_{n t}\right)$ are contained in the closed $\varepsilon_{n}$-neighborhood $N_{\varepsilon_{n}}\left(a_{0 t}\right)$ of

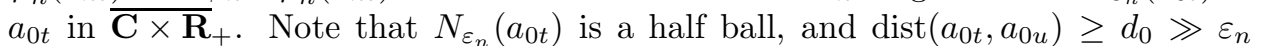
for $t \neq u$. The subset $A\left(\varepsilon_{n}\right)$ of $\operatorname{Isom}^{+}\left(\mathbf{H}^{3}\right)$ consisting of Möbius transformations $\eta$ with $\eta\left(N_{\varepsilon_{n}}\left(a_{0 t}\right)\right) \cap N_{\varepsilon_{n}}\left(a_{0 t}\right) \neq \emptyset$ for $t=1,2,3$ is compact, and $\bigcap_{n=1}^{\infty} A\left(\varepsilon_{n}\right)=\{\operatorname{Id}\}$. Then, $\psi_{n} \in A\left(\varepsilon_{n}\right)$ converges to the identity in $\operatorname{Isom}^{+}\left(\mathbf{H}^{3}\right)$, or equivalently $\left(\varphi_{n}\right)_{*}\left(\gamma_{i}\right)$ converges to $\gamma_{0 i}$ in $\operatorname{Isom}^{+}\left(\mathbf{H}^{3}\right)$. This shows (3.7).

By (3.7), one can choose $c_{n i}$ 's $(n \in \mathbf{N})$ which are equicontinuous, and with

$$
L=\sup \left\{\operatorname{length}_{N_{n}}\left(c_{n i}\right) ; i=1, \ldots, k, n \in \mathbf{N}\right\}<\infty .
$$

For all $n \in \mathbf{N}$ with $\varepsilon_{n}<\varepsilon_{0}$ and $\operatorname{dist}_{N_{n}}\left(\partial N_{n, \operatorname{thin}\left(\varepsilon_{0}\right)}, \partial N_{n, \operatorname{thin}\left(\varepsilon_{n}\right)}\right)>2 L, c_{n 1} \cup$ $\cdots \cup c_{n k}$ is disjoint from $N_{n, \operatorname{thin}\left(\varepsilon_{n}\right)}$. Otherwise, $c_{n 1} \cup \cdots \cup c_{n k}$ would be contained in $N_{n, \operatorname{thin}\left(\varepsilon_{0}\right)}$, and hence $\left(\varphi_{n}\right)_{*}\left(\gamma_{n i}\right)(i=1, \ldots, k)$ would generate a cyclic group. This contradicts that the sequences $\left\{\left(\varphi_{n}\right)_{*}\left(\gamma_{n i}\right)\right\}$ converge to $\gamma_{0 i}$ 's which generate non-elementary group $\pi_{1}(X)$. Since $h_{n}: N_{n, \operatorname{thick}\left(\varepsilon_{n}\right)} \longrightarrow Y_{\operatorname{thick}\left(\varepsilon_{n}\right)}$ is a $K_{n}$-quasiisometry with $K_{n} \searrow 1$ for $i=1, \ldots, k$, again by passing to a subsequence, we may assume that each $\left\{h_{n} \circ c_{n i}\right\}_{n=1}^{\infty}$ converges uniformly to a curve $c_{\infty i}$ in $Y$. This shows that $h_{n} \circ c_{n i}$ is homotopic to $c_{\infty i}$ in $Y$ for all sufficiently large $n \in \mathbf{N}$. Let $\gamma_{\infty i}$ be the element of $\pi_{1}\left(Y, y_{\infty}\right)$ represented by $c_{\infty i}$, and $\Gamma_{\infty}$ the subgroup of $\pi_{1}\left(Y, y_{\infty}\right)$ generated by $\gamma_{\infty 1}, \ldots, \gamma_{\infty k}$, where $y_{\infty}=\lim _{n \rightarrow \infty} h_{n}\left(y_{n}\right)$. Consider the universal covering $q_{\infty}: \mathbf{H}^{3} \longrightarrow Y$ of $Y$. Again by the $K_{n}$-quasi-isometricity of $h_{n}$ with $K_{n} \searrow 1$, each $\left\{\left(\varphi_{n}\right)_{*}\left(\gamma_{n i}\right)\right\}$ converges to $\gamma_{\infty i}$ in $\operatorname{Isom}^{+}\left(\mathbf{H}^{3}\right)$, where $\gamma_{\infty i} \in \pi_{1}\left(Y, y_{\infty}\right)$ is regarded as a covering transformation on $\mathbf{H}^{3}$ with respect to a suitable coordinate on $\mathbf{H}^{3}$ with $q_{\infty}(0,1)=y_{\infty}$. This shows that $\gamma_{0 i}$ is equal to $\gamma_{\infty i}$ as elements of $\operatorname{Isom}^{+}\left(\mathbf{H}^{3}\right)$ for $i=1,2, \ldots, k$, and hence in particular $\pi_{1}\left(X, x_{0}^{\prime}\right)=\Gamma_{\infty}$ for $x_{0}^{\prime}=p\left(\alpha_{0} \widetilde{x}_{0}\right)$. Since $\operatorname{Vol}(X)=\operatorname{Vol}(Y), \Gamma_{\infty}$ is equal to $\pi_{1}\left(Y, y_{\infty}\right)$. It follows that there exists an isometry $\psi: X \longrightarrow Y$ with $\psi_{*}\left(\gamma_{0 i}\right)=\gamma_{\infty i}$ for $i=1,2, \ldots, k$. By our construction of $\psi$, the homeomorphism

$$
j_{n}=h_{n}^{-1} \circ\left(\psi \mid X_{\operatorname{thick}\left(\varepsilon_{n}\right)}\right) \circ g_{n}^{-1}: M_{n, \operatorname{thick}\left(\varepsilon_{n}\right)} \longrightarrow N_{n, \operatorname{thick}\left(\varepsilon_{n}\right)} \subset N_{n}
$$

is homotopic to $f_{n} \mid M_{n, \operatorname{thick}\left(\varepsilon_{n}\right)}: M_{n, \operatorname{thick}\left(\varepsilon_{n}\right)} \longrightarrow N_{n}$. Let $m_{V}$ be a meridian of a component $V$ of $M_{n, \operatorname{thin}\left(\varepsilon_{n}\right)}$. Since $j_{n}\left(m_{V}\right) \simeq f_{n}\left(m_{V}\right)$ is contractible in $N_{n}$, $j_{n}\left(m_{V}\right)$ is a meridian of the component of $N_{n, \operatorname{thin}\left(\varepsilon_{n}\right)}$ bounded by $j_{n}(\partial V)$. Then, $j_{n}$ would be extended to a homeomorphism $\widehat{j}_{n}: M_{n} \longrightarrow N_{n}$ homotopic to $f_{n}$, a contradiction. This completes our reduction to absurdity and hence the proof of Theorem 2.

Remark 1. In the special case of $M_{n}=X, N_{n}=Y$ and $f_{n}=f$ for all $n \in \mathbf{N}$, the map $\psi: X \longrightarrow Y$ above is an isometry homotopic to $f: X \longrightarrow Y$. Thus, we have given a new proof of Gromov-Thurston's Rigidity Theorem without using the radially extended map $\widetilde{f}_{\infty}: S_{\infty}^{2} \longrightarrow S_{\infty}^{2}$.

We will now present the example which shows that Theorem 2 is false for maps of higher degree.

Example 1. Let $K$ be a figure-eight knot in $S^{3}$, and let $p: M \longrightarrow S^{3}$ be a $d_{0}$-fold, cyclic branched covering of $S^{3}$ branched over $K$ for $d_{0} \in \mathbf{N}$ with $d_{0}>1$. The complement $S^{3}-K$ has a complete hyperbolic structure with $\operatorname{Vol}\left(S^{3}-K\right)=2 \mathbf{v}_{3}$. Consider a regular neighborhood $V$ of $K$ in $S^{3}$, and the preimage $\widetilde{V}=p^{-1}(V)$. Let $\mu, \lambda \subset \partial V$ be a meridian and a longitude for $\left(S^{3}, V\right)$ respectively. Then, $\widetilde{\mu}=p^{-1}(\mu)$ is a meridian of the solid torus $\widetilde{V}$. Take a component $\widetilde{\lambda}$ of $p^{-1}(\lambda)$. 
The homomorphism $(p \mid \partial \widetilde{V})_{*}: H_{1}(\partial \widetilde{V} ; \mathbf{Z}) \longrightarrow H_{1}(\partial V ; \mathbf{Z})$ maps $[\widetilde{\mu}]$ to $d_{0}[\mu]$, and $[\widetilde{\lambda}]$ to $[\lambda]$. For any $n \in \mathbf{N}$, let $s_{n}$ be a simple loop in $\partial V$ representing $[\mu]+n[\lambda]$ in $H_{1}(\partial V ; \mathbf{Z})$. Then, $\widetilde{s}_{n}=p^{-1}\left(s_{n}\right)$ is also a simple loop in $\partial \widetilde{V}$ representing $[\widetilde{\mu}]+d_{0} n[\tilde{\lambda}]$ in $H_{1}(\partial \widetilde{V} ; \mathbf{Z})$. Let $M_{n}$ be the 3 -manifold obtained by attaching a solid torus $\widetilde{V}_{0}$ to $M-\operatorname{int} \widetilde{V}$ by a homeomorphism $\partial \widetilde{V}_{0} \longrightarrow \partial \widetilde{V}$ which maps a meridian of $\partial \widetilde{V}_{0}$ onto $\widetilde{s}_{n}$. Let $N_{n}$ be the 3-manifold obtained by attaching a solid torus $V_{0}$ to $S^{3}-\operatorname{int} V$ by a homeomorphism $\partial V_{0} \longrightarrow \partial V$ which maps a meridian of $\partial V_{0}$ onto $s_{n}$. Then, the restriction $p \mid(M-\operatorname{int} \widetilde{V}): M-\operatorname{int} \widetilde{V} \longrightarrow S^{3}-\operatorname{int} V$ is extended to a $d_{0}$-fold, cyclic branched covering $f_{n}: M_{n} \longrightarrow N_{n}$ branched over the core $\Sigma_{n}$ of $V_{0}$. By the Hyperbolic Dehn Surgery Theorem [20, Theorem 5.9], for all sufficiently large $n \in \mathbf{N}$, both $M_{n}$ and $N_{n}$ have hyperbolic structures, and $\lim _{n \rightarrow \infty} \operatorname{Vol}\left(M_{n}\right)=$ $d_{0} \lim _{n \rightarrow \infty} \operatorname{Vol}\left(N_{n}\right)=2 d_{0} \mathbf{v}_{3}$. For any point $x_{0} \in \Sigma_{n}$, the preimage $f_{n}^{-1}\left(x_{0}\right)$ consists of a single point $\widetilde{x}_{0}$ in $M_{n}$. Then, any simple closed loop $l$ in $N_{n}$ with $l \cap \Sigma_{n}=\left\{x_{0}\right\}$ is covered homeomorphically by a loop $\widetilde{l}$ in $M_{n}$ with $\widetilde{l} \cap f_{n}^{-1}\left(\Sigma_{n}\right)=\left\{\widetilde{x}_{0}\right\}$. This shows that $\left(f_{n}\right)_{*}: \pi_{1}\left(M_{n}\right) \longrightarrow \pi_{1}\left(N_{n}\right)$ is surjective, and hence $f_{n}$ is not homotopic to an (unbranched) $d_{0}$-fold covering.

Proof of Corollary 1. We suppose that there are no constants $c>0$ satisfying the property (0.2) in Corollary 1. Then, for any $n \in \mathbf{N}$, there would exist a degree-one, but non-homotopy equivalence map $f_{n}: M_{n} \longrightarrow N_{n}$ between closed, connected, hyperbolic 3-manifolds with $\operatorname{Vol}\left(M_{n}\right) \leq V$ and $(1-1 / n) \operatorname{Vol}\left(M_{n}\right) \leq \operatorname{Vol}\left(N_{n}\right)$. Since $\operatorname{Vol}\left(N_{n}\right) \leq \operatorname{Vol}\left(M_{n}\right)$, there exist subsequences $\left\{M_{n_{i}}\right\},\left\{N_{n_{i}}\right\}$ with

$$
\lim _{n_{i} \rightarrow \infty} \operatorname{Vol}\left(M_{n_{i}}\right)=\lim _{n_{i} \rightarrow \infty} \operatorname{Vol}\left(N_{n_{i}}\right) \leq V .
$$

This contradicts Theorem 2, and hence we have a constant $c>0$ satisfying (0.2).

Proof of Corollary 2. Consider any ascending sequence

$$
M_{0} \stackrel{f_{1}}{\longleftarrow} M_{1} \stackrel{f_{2}}{\longleftarrow} M_{2} \stackrel{f_{3}}{\longleftarrow} \ldots \stackrel{f_{n}}{\longleftarrow} M_{n}
$$

of non-homotopy equivalence, degree-one maps between closed, hyperbolic 3-manifolds. If $\operatorname{Vol}\left(M_{n}\right) \leq V$, then Corollary 1 implies

$$
(1-c(V))^{n} \operatorname{Vol}\left(M_{n}\right)>\operatorname{Vol}\left(M_{0}\right) \geq \mathbf{v}_{0},
$$

where $\mathbf{v}_{0}>0$ is the smallest volume of hyperbolic 3-manifolds, see [20 Chapter 6] for the existence of $\mathbf{v}_{0}$. It follows that

$$
n<\frac{\log \mathbf{v}_{0}-\log V}{\log (1-c(V))}
$$

Thus, the smallest integer $n_{0}$ not less than $\left(\log \mathbf{v}_{0}-\log V\right) / \log (1-c(V))$ satisfies the property (0.3) of Corollary 2.

\section{Divergent SEquences of Degree-One maps}

In this section, we will prove Theorem 1 by using the argument similar to that in Fujii-Soma [4].

Let $A$ be a compact, connected, hyperbolic 3-manifold such that the boundary $\partial A$ consists of two totally geodesic components $\Sigma, \Sigma^{\prime}$. Moreover, we suppose that $A$ admits an orientation-reversing, isometric involution $\tau: A \longrightarrow A$ with $\tau(\Sigma)=\Sigma^{\prime}$, and contains a two-sided, closed surface $F$ such that $A-F$ is connected, for example see [4] Lemma 1] for the existence of such a 3-manifold $A$. A connected sum $A^{\prime}=$ $A \# S^{1} \times S^{2}$ is obtained from $A-\operatorname{int} B_{0}$ and $S^{1} \times S^{2}-\operatorname{int} B_{1}$ by attaching $\partial B_{1}$ to $\partial B_{0}$ 
by an orientation-reversing homeomorphism, where $B_{0}, B_{1}$ are 3-balls embedded in $\operatorname{int} A$ and $S^{1} \times S^{2}$ respectively. One can choose $B_{1}$ so that $S^{1} \times\{x\} \cap B_{1}=\emptyset$ for some $x \in S^{2}$. For any $m \in \mathbf{N}$, let $\varphi_{m}: A^{\prime} \longrightarrow A$ be a proper, degree-one map such that $\varphi_{m} \mid\left(A-\operatorname{int} B_{0}\right)$ is the identity and the algebraic intersection number $\left[\left(\varphi_{m}\right)_{*}\left(S^{1} \times\{x\}\right)\right] \cdot[F]=m$. By Theorem 6.1 in Myers [14 together with Thurston's Uniformization Theorem [21] and Mostow's Rigidity Theorem, there exists a nullhomotopic knot $K$ in $A^{\prime}$ with $K \cap S^{1} \times\{x\}=\emptyset$ such that $A^{\prime}-K$ admits a complete, hyperbolic structure with totally geodesic boundary. By the Hyperbolic Dehn Surgery Theorem, one can obtain a compact hyperbolic 3-manifold $\widehat{A}$ with totally geodesic boundary by Dehn surgery on $A^{\prime}$ along $K$. By Proposition 3.2 in Boileau-Wang [1], there exists a degree-one map $\widehat{\varphi}: \widehat{A} \longrightarrow A^{\prime}$ extending the identity of the complement of a small neighborhood of $K$ in $A^{\prime}$. Boileau and Wang considered only the case where 3 -manifolds containing null-homotopic knots are irreducible. However, their proof holds even in the non-irreducible case without any alterations. Thus, we have a degree-one map $\psi_{m}=\varphi_{m} \circ \widehat{\varphi}: \widehat{A} \longrightarrow A$ such that $\psi_{m} \mid \partial \widehat{A}: \partial \widehat{A} \longrightarrow \partial A$ is the identity of $\Sigma \cup \Sigma^{\prime}$. Let $A_{1}, \ldots, A_{2 n}$ be $2 n$ copies of $A$, and let $\eta_{i}: A \longrightarrow A_{i}$ be the identity for $i=1,2, \ldots, 2 n$. We set $\eta_{i}(\Sigma)=\Sigma_{i}$ and $\eta_{i}\left(\Sigma^{\prime}\right)=\Sigma_{i}^{\prime}$. Construct the "long" hyperbolic 3-manifold $C_{n}$ from $A_{1}, A_{2}, \ldots, A_{2 n}$ by connecting $A_{i}$ with $A_{i+1}$ by the orientation-reversing isometry $\left(\eta_{i+1} \mid \Sigma\right) \circ\left(\tau \mid \Sigma^{\prime}\right) \circ$ $\left(\eta_{i}^{-1} \mid \Sigma_{i}^{\prime}\right): \Sigma_{i}^{\prime} \longrightarrow \Sigma_{i+1}$ for $i=1,2, \ldots, 2 n-1$. Let $M_{n}$ (resp. $\left.N_{n}\right)$ be a closed 3manifold obtained from $\widehat{A}$ (resp. $A$ ) and $C_{n}$ by identifying $\Sigma \cup \Sigma^{\prime}$ and $\partial C_{n}=$ $\Sigma_{1} \cup \Sigma_{2 n}^{\prime}$ by the orientation-reversing homeomorphisms $\left(\eta_{1} \mid \Sigma\right) \circ\left(\tau \mid \Sigma^{\prime}\right): \Sigma^{\prime} \longrightarrow \Sigma_{1}$ and $\left(\eta_{2 n} \mid \Sigma^{\prime}\right) \circ(\tau \mid \Sigma): \Sigma \longrightarrow \Sigma_{2 n}^{\prime}$. Note that $N_{n}$ admits the hyperbolic structure extending those on $A$ and $C_{n}$. Though $M_{n}$ also admits a hyperbolic structure by Thurston's Uniformization Theorem, the structure is not in general the extention of those on $\widehat{A}$ and $C_{n}$.

Now, we will show that, for a sufficiently large $n \in \mathbf{N}, M=M_{n}$ and $N=N_{n}$ satisfy the property $(0.1)$ of Theorem 1 .

Proof of Theorem 1. According to Freedman-Hass-Scott [3], we may assume that each $\Sigma_{i}^{\prime}=\Sigma_{i+1}$ is a least area surface in $M_{n}$ among its homotopy class. Then, $M_{n}$ is divided by these least area surfaces into the pieces $\widehat{A}^{(n)}, A_{1}^{(n)}, \ldots, A_{2 n}^{(n)}$ corresponding to $\widehat{A}, A_{1}, \ldots, A_{2 n}$ respectively. Then, one can show the following (4.1) and (4.2) by the argument quite similar to that in [4], which is based on McMullen's work in [1] and 12 .

$$
\sup _{n \in \mathbf{N}}\left\{\max \left\{\operatorname{Vol}\left(\widehat{A}^{(n)}\right), \operatorname{Vol}\left(A_{1}^{(n)}\right), \ldots, \operatorname{Vol}\left(A_{2 n}^{(n)}\right)\right\}\right\}=L<\infty .
$$

(4.2) Suppose that $0<\eta<1$ is arbitrarily given. Then, there exists $n_{0}(\eta) \in \mathbf{N}$ such that

$$
1-\eta<\frac{\operatorname{Vol}\left(A_{j}^{(n)}\right)}{\operatorname{Vol}(A)}<1+\eta
$$

for any $n \in \mathbf{N}$ with $n>n_{0}$ and any $j=n_{0}+1, n_{0}+2, \ldots, 2 n-n_{0}-1,2 n-n_{0}$.

Intuitively, the (4.1) implies that the induced metrics on $\widehat{A}^{(n)}$ and $A_{i}^{(n)}$ from that on $M_{n}$ remain in "bounded regions" independent of $n$. In fact, (4.1) can be proved also by using the two facts that (i) the topological type of the union $B$ of $\widehat{A}^{(n)}$ or $A_{i}^{(n)}$ and its adjacent pieces (e.g. $B=A_{1}^{(n)} \cup \widehat{A}^{(n)} \cup A_{2 n}^{(2 n)}$ ) is independent of $n$, and (ii) the closure of the set of all complete, geometrically finite, hyperbolic 
structures on $\operatorname{int} B$ is compact in the algebraic deformation space by Thurston [22]. (4.2) is derived from the fact that, except for the first and last $n_{0}$ pieces, all other $A_{i}^{(n)}$ 's are well and uniformly approximated by $A$, see [4, §3] for details. By (4.1) and (4.2), for any $n \in \mathbf{N}$ with $n>n_{0}$, we have

$$
2\left(n-n_{0}\right)(1-\eta) \operatorname{Vol}(A)<\operatorname{Vol}\left(M_{n}\right)<\left(2 n_{0}+1\right) L+2\left(n-n_{0}\right)(1+\eta) \operatorname{Vol}(A) .
$$

Since $\operatorname{Vol}\left(N_{n}\right)=(2 n+1) \operatorname{Vol}(A)$, we have $\lim _{n \rightarrow \infty} \operatorname{Vol}\left(M_{n}\right) / \operatorname{Vol}\left(N_{n}\right)=1$. For any $\varepsilon>0$, there exists $n_{1} \in \mathbf{N}$ with $(1-\varepsilon) \operatorname{Vol}\left(M_{n_{1}}\right) \leq \operatorname{Vol}\left(N_{n_{1}}\right)$. Set $M=M_{n_{1}}$, $N=N_{n_{1}}$, and let $f_{m}: M \longrightarrow N$ be the degree-one map such that $f_{m} \mid \widehat{A}=\psi_{m}$ and $f_{m} \mid A_{i}: A_{i} \longrightarrow A_{i}\left(i=1,2, \ldots, 2 n_{1}\right)$ is the identity of $A$. For a simple loop $l$ in $M$ with $\widehat{\varphi}(l)=S^{1} \times\{x\}$, we have

$$
\left[\left(f_{m}\right)_{*}(l)\right] \cdot[F]=\left[\left(\varphi_{m}\right)_{*}\left(S^{1} \times\{x\}\right)\right] \cdot[F]=m .
$$

It follows that

$$
\lim _{m \rightarrow \infty} \inf \left\{\delta(g) ; g \text { is a } C^{1} \text {-map homotopic to } f_{m}\right\}=\infty .
$$

This completes the proof.

\section{REFERENCES}

1. M. Boileau and S. Wang, Non-zero degree maps and surface bundles over $S^{1}$, J. Differential Geom. 43 (1996), 789-806. MR 98g:57023

2. N. Dunfield, Cyclic surgery, degree of maps of character curves, and volume rigidity for hyperbolic manifolds, Invent. Math. 136 (1999), 623-657. MR 2000d:57022

3. M. Freedman, J. Hass and P. Scott, Least area incompressible surfaces in 3-manifolds, Invent. Math. 71 (1983), 609-647. MR 85e:57012

4. M. Fujii and T. Soma, Totally geodesic boundaries are dense in the moduli space, J. Math. Soc. Japan 49 (1997), 589-601. MR 99b:57029

5. W. Goldman, Discontinuous groups and the Euler class, Ph. D. Thesis, U. C. Berkeley (1980).

6. C. Hayat-Legrand, S. Wang and H. Zieschang, Degree-one maps onto lens spaces, Pacific J. Math. 176 (1996), 19-32. MR 98b:57030

7. C. Hayat-Legrand, S. Wang and H. Zieschang, Minimal Seifert manifolds, Math. Ann. 308 (1997), 673-700. MR 98i:57029

8. J. Hempel, 3-manifolds, Ann. of Math. Studies 86, Princeton Univ. Press, Princeton N.J. (1976). MR 54:3702

9. W. Jaco, Lectures on three-manifold topology, C.B.M.S. Regional Conf. Ser. in Math. no. 43, Amer. Math. Soc., Providence, R.I. (1980). MR 81k:57009

10. R. Kirby, Problems in low-dimensional topology, Geometric Topology (W.H. Kazez ed.), AMS/IP Studies in Advanced Mathematics vol. 2, Part 2, Amer. Math. Soc. and International Press (1997), 35-473. MR 98f:57001

11. C. McMullen, Amenability, Poincaré series and quasiconformal maps, Invent. Math. 97 (1989), 95-127. MR 90e:30048

12. C. McMullen, Iteration on Teichmüller space, Invent. Math. 99 (1990), 425-454. MR 91a:57008

13. G. Mostow, Quasi-conformal mappings in n-space and the rigidity of hyperbolic space forms, Publ. Math. I.H.E.S. 34 (1968), 53-104. MR 38:4679

14. R. Myers, Simple knots in compact, orientable 3-manifolds, Trans. Amer. Math. Soc. 273 (1982), 75-91. MR 83h:57018

15. A. Reid, S. Wang and Q. Zhou, Generalized Hopfian property, minimal Haken manifold, and J. Simon's conjecture for 3-manifold groups, preprint.

16. Y. Rong, Degree one maps between geometric 3-manifolds, Trans. Amer. Math. Soc. 322 (1992), 411-436. MR 92j:57007

17. T. Soma, Bounded cohomology of closed surfaces, Topology 36 (1997), 1221-1246. MR 99a:57011

18. T. Soma, Non-zero degree maps to hyperbolic 3-manifolds, J. Differential Geom. 49 (1998), 517-546. MR 2000b:57034 
19. T. Soma, Sequences of degree-one maps between geometric 3-manifolds, Math. Ann. 316 (2000), 733-742. MR 2001b:57039

20. W. Thurston, The geometry and topology of 3-manifolds, Lecture Notes, Princeton Univ., Princeton (1978), http://www.msri.org/publications/gt3m/

21. W. Thurston, Three dimensional manifolds, Kleinian groups and hyperbolic geometry, Bull. Amer. Math. Soc. 6 (1982), 357-381. MR 83h:57019

22. W. Thurston, Hyperbolic structures on 3-manifolds I: Deformation of acylindrical manifolds, Ann. of Math. 124 (1986), 203-246. MR 88g:57014

23. H. C. Wang, Topics on totally discontinuous groups, In: Symmetric Spaces (W. Boothby and G. Weiss eds.) Pure and Appl. Math. 8, Marcel Dekker, New York (1972), 459-487. MR 54:2879

24. S. Wang and Q. Zhou, Any 3-manifold 1-dominates at most finitely many geometric 3manifolds, preprint.

25. A. Zastrow, On the (non)-coincidence of Milnor-Thurston homology theory with singular homology theory, Pacific J. Math. 186 (1998), 369-396. MR 2000a:55008

Department of Mathematical Sciences, College of Science and Engineering, Tokyo Denki University, Hatoyama-machi, Saitama-ken 350-0394, Japan

E-mail address: soma@r.dendai.ac.jp 